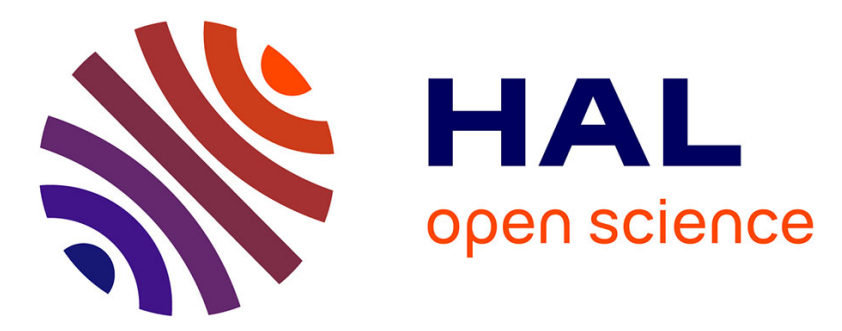

\title{
Mieux valoriser l'information contenue dans les carnets d'agnelage pour évaluer des performances et des carrières de reproduction en élevage ovin-viande
}

\author{
J. Lasseur, E. Landais
}

\section{> To cite this version:}

J. Lasseur, E. Landais. Mieux valoriser l'information contenue dans les carnets d'agnelage pour évaluer des performances et des carrières de reproduction en élevage ovin-viande. Productions Animales, 1992, 5 (1), pp.43-58. hal-00895963

\section{HAL Id: hal-00895963 https://hal.science/hal-00895963}

Submitted on 1 Jan 1992

HAL is a multi-disciplinary open access archive for the deposit and dissemination of scientific research documents, whether they are published or not. The documents may come from teaching and research institutions in France or abroad, or from public or private research centers.
L'archive ouverte pluridisciplinaire HAL, est destinée au dépôt et à la diffusion de documents scientifiques de niveau recherche, publiés ou non, émanant des établissements d'enseignement et de recherche français ou étrangers, des laboratoires publics ou privés. 
INRA Prod. Anim.,

$1992,5(1), 43-58$

\section{J. LASSEUR et E. LANDAIS *}

INRA SAD, Domaine Saint-Paul, 84143 Montfavet Cédex

* INRA SAD, Route de Saint-Cyr, 78000 Versailles
Mieux valoriser

l'information

contenue dans les

carnets d'agnelage

pour évaluer des

performances et des

carrières de

reproduction en

élevage ovin-viande

Contrairement à l'expérimentation, qui consiste à créer des situations particulières et à organiser le recueil des données de façon à pouvoir répondre à une question formulée a priori, l'étude des systèmes de production en ferme doit s'adapter aux situations existantes, et utiliser au mieux l'information disponible plutôt que de proposer d'emblée des contrôles ou des enregistrements nouveaux, qui représentent des contraintes supplémentaires pour les exploitants. Ceci conduit à développer des méthodes d'investigation spécifiques, qui valorisent au maximum les données existantes et répondent aux besoins concrets des producteurs. Dans cette optique, cet article consacré aux systèmes d'élevage ovin-viande s'intéresse à la valorisation, généralement très partielle, de l'information contenue dans un document largement utilisé par les éleveurs français : le carnet d'agnelage.

Le carnet d'agnelage est utilisé par les éleveurs comme "carnet de bord" pour le suivi des périodes de mise-bas et de la phase d'allaitement : il joue alors un rôle important pour l'allotement et la

\section{Résumé}

S'appuyant sur l'exemple de l'étude d'une exploitation d'élevage ovin-viande du Sud de la France, les auteurs proposent une méthode rétrospective d'analyse des performances de reproduction utilisant l'information contenue dans les carnets d'agnelage, documents largement utilisés par les éleveurs français.

Différents modes d'organisation de cette information permettent de développer trois volets d'étude complémentaires :

- l'analyse de l'évolution saisonnière et interannuelle des performances de reproduction à l'échelle du troupeau ;

- l'analyse de la variabilité intra-troupeau des performances individuelles (précocité, aptitude au désaisonnement, fertilité, régularité, prolificité, longévité), qui débouche sur une caractérisation globale des carrières des brebis ;

- l'analyse combinée, qui associe les deux volets d'étude précédents, et qui permet de rendre compte des rythmes de reproduction, lesquels résultent des interactions entre les variations du milieu d'élevage et les comportements individuels des animaux.

Le développement de ce type d'étude et son application à des systèmes d'élevage variés pourrait permettre de déboucher sur des conclusions opératoires en termes de conduite des troupeaux et peut-être de choix du matériel génétique. conduite des troupeaux. Son utilisation est toutefois bien souvent limitée à cette fonction. Sa valorisation par les organismes de développement se limite généralement à la réalisation d'un bilan de campagne de reproduction : calcul de la prolificité et du taux de mise-bas moyen, si l'enregistrement de l'inventaire troupeau a été réalisé (ce qui est loin d'être le cas général).

Deux voies d'analyse complémentaires peuvent cependant être envisagées, même en l'absence de contrôles d'inventaire :

- la première vise à caractériser globalement les performances de reproduction réalisées par le troupeau, dans le cadre d'analyses menées à l'échelle de périodes spécifiées : sessions de reproduction ou exercices annuels. Cette méthode permet de réaliser des comparaisons entre périodes ou entre années (pour un même troupeau ou un même ensemble de troupeaux), ainsi que des comparaison entre troupeaux.

- la seconde vise à caractériser les performances individuelles, dans le cadre d'analyses prenant en compte les carrières des femelles reproductrices. Cette approche renvoie à l'analyse des rythmes biologiques de reproduction, et rend compte de la variabilité des performances intra-troupeau. Nous 
l'avons utilisée pour étudier des systèmes d'élevage relativement extensif, où l'alimentation est peu maîtrisée : de nombreux auteurs ont montré les conséquences à court terme (Haresign 1983) ou à long terme (Gunn 1977) des périodes de restriction alimentaire sur les performances de reproduction. Dans ces conditions, les éleveurs attendent de leurs animaux qu'ils tamponnent en partie l'effet de la variabilité saisonnière et interannuelle des ressources exploitées, variabilité due principalement aux aléas climatiques (Vallerand et Santucci 1987). Certains animaux présentent ainsi des rythmes de reproduction irréguliers, ce dont les éleveurs semblent s'accomoder, dans la mesure où le coût marginal d'un animal temporairement inproductif est relativement faible dans ce type de systèmes de production. Les observations conduites à l'échelle des carrières animales donnent souvent lieu à des bilans permettant la comparaison de divers "traitements" : mise-bas précoce versus mise-bas tardive (Baker et al 1978), origines génétiques différentes (Dalton et al 1976), etc. Mais l'étude des carrières de reproduction permet aussi d'affiner la connaissance de ces phénomènes d'adaptation et de préciser les manières d'en tirer parti au mieux. Elle rejoint les réflexions des généticiens relatives à la rusticité des races locales (Flamant et al 1979), et à leur adaptation à des systèmes d'élevage particuliers, valorisant leurs aptitudes propres (Vallerand 1979 et 1988). L'une des questions posées à ce sujet est la suivante : la longévité des femelles reproductrices peut-elle être considérée comme un bon indicateur de leur adaptation au système d'élevage auquel elles appartiennent?

\section{Le carnet d'agnelage}

Le carnet d'agnelage, diffusé principalement par IITOVIC, est dans sa conception le document d'enregistrement de base des informations concernant le troupeau. Ce livret permet, pour chaque mise-bas, l'enregistrement : du numéro de la brebis, du nombre d'agneaux nés, de leur sexe, de la date de l'événement ainsi que du numéro d'identification du bélier (lorsqu'il est connu) et d'informations "libres" concernant le déroulement de la période post-partum (vitalité des agneaux, comportement de la mère, adoption éventuelle, etc.). Est également prévu l'enregistrement d'informations sanitaires (vaccinations, traitement antiparasitaires, etc.), de l'inventaire du troupeau, de la date de début et de fin de chaque session $\left({ }^{*}\right)$ de lutte, de l'alimentation et de la date de quelques événements ponctuant la campagne d'élevage (tonte, rentrée en bergerie et mise à l'herbe, désinfection des locaux), ainsi que la réalisation d'un bilan technique et sanitaire de la campagne.

Dans la pratique, le carnet d'agnelage est effectivement présent dans de nombreux élevages, car il fait partie des documents dont la tenue est contractuellement obligatoire pour les bénéficiaires d'appui technique dans le cadre du plan de développement de l'élevage ovin et pour les adhérents à des groupements de producteurs.

* Pour éviter les ambiguités dues aux multiples usages du terme de "période", nous employons le terme de "session" pour désigner spécifiquement les périodes de lutte et de mise-bas.

\section{1 / Conduite de l'étude}

La méthode que nous proposons sera présentée à partir de l'étude d'un exemple concret, volontairement choisi parmi les systèmes d'élevage présentant plusieurs sessions de mise-bas annuelles, qui sont les plus compliqués à analyser (Vissac 1988). Il s'agit du troupeau ovin d'une exploitation de 35 ha de SAU située en zone de moyenne montagne méditerranéenne dans le département des Alpes de Haute-Provence. Ce troupeau, étudié du début de l'année 1977 à la fin de l'année 1989, regroupait en moyenne, durant cette période, 250 brebis de race Préalpes du Sud dont la productivité moyenne s'est établie à 1,2 agneau mis bas par brebis et par an (tableau 5). De mai à janvier l'alimentation provient exclusivement du pâturage (parcours, prairies artificielles, chaumes de céréales). Durant la période hivernale (févriermars-avril), les animaux sont cloîtrés en bergerie et reçoivent une alimentation à base de foin. La complémentation, constituée de céréales, est réservée aux brebis allaitantes.

Les agneaux destinés à la boucherie sont élevés en bergerie, quelle que soit la saison, et abattus à partir de l'âge de trois mois, à un poids vif compris entre 30 et $35 \mathrm{~kg}$.

Trois sessions de mise-bas de durée sensiblement équivalente (environ 45 jours) se succèdent au cours de l'année à partir du 15 décembre (misebas d'hiver) puis du 10 mars (mise-bas de printemps) et enfin du 15 août (mise-bas d'automne), ces dates étant fixées par l'exploitant afin d'aménager au mieux son calendrier de travail. Elles correspondent respectivement à des luttes naturelles centrées sur les mois d'août, de novembre et d'avril. Durant ces périodes, les béliers (provenant d'élevages locaux) sont introduits dans le troupeau sans aucun tri des femelles. Les agnelles de remplacement (qui sont toutes issues du troupeau ou, jusqu'en 1982, d'un troupeau voisin ayant adopté le même rythme de reproduction) sont choisies uniquement parmi celles qui sont issues de la lutte d'été, l'éleveur considérant que cela simplifie la conduite du troupeau. Bien qu'elles ne soient pas séparées des femelles adultes lors de périodes de lutte, les premières saillies fécondantes n'interviennent jamais avant l'été qui précède leur premier anniversaire. Les brebis ne sont réformées que lorsqu'elles se trouvent dans l'incapacité de produire : troubles pathologiques graves, infécondité, chute des incisives.

\section{1 / Nature des données}

Les carnets d'agnelage représentent l'unique source d'informations. Les renseignements qu'ils contiennent sont enregistrés au jour le jour par l'éleveur. Chaque ligne correspond à une mise-bas. Nous avons utilisé trois données : l'identification de la brebis (numéro de son tatouage auriculaire, comprenant le quantième de son année de naissance et son numéro d'ordre individuel), la date de la mise-bas et le nombre d'agneaux nés (les avortements ne sont pas signalés ; les agneaux mortsnés ou morts dans les 48 heures suivant la naissance ne sont pas régulièrement distingués). L'information traitée est donc rudimentaire. Elle est cependant représentative de celle qui est dis- 
ponible dans de très nombreux élevages ovins français, ce qui fait tout son intérêt, et justifie la tentative de valorisation présentée ici.

\section{2 / Traitement des données}

L'information recueillie sur le déroulement des sessions de mise-bas est en fait utilisée pour analyser les résultats des sessions de lutte qui ont eu lieu cinq mois auparavant, que nous considérons comme les événements les plus déterminants du cycle de reproduction.

Le principe du traitement des données qui a été adopté consiste à "coller" aussi étroitement que possible à la nature des informations de base, en constituant deux matrices (R1) et (R2) dont chaque ligne représente une femelle reproductrice, chaque session de mise-bas étant représentée par une colonne contenant un chiffre :

- 0 si aucune mise-bas n'est signalée pour la brebis considérée pour la période en question ;

- 1 ou 2 si une mise-bas est signalée : le chiffre 1 indique une portée simple, le chiffre 2 , une portée double (aucune portée triple n'a été enregistrée, mais cette éventualité ne soulèverait aucun problème particulier).

\section{a / Analyse des performances à l'échelle du troupeau}

Pour cette analyse, chaque colonne représente une session de mise-bas identifiée par référence au calendrier. La colonne de gauche représente ainsi la session de mise-bas centrée sur le mois de janvier 1977, celle de droite, la session centrée sur le mois de janvier 1989. Par construction, les évènements de reproduction d'une même colonne sont donc contemporains.

Le nombre de lignes de cette matrice de départ est égal au nombre des brebis qui ont été signalées comme ayant mis bas au moins une fois dans le troupeau étudié sur la période considérée, soit $\mathrm{N}=536$. Moyennant un certain nombre de conven- tions (voir encadré), nous parvenons ainsi à la configuration de la matrice (R1), illustrée par le tableau 1.

La prolificité $(\pi)$ est aisément calculée grâce à cette matrice, à partir de la taille des portées mises-bas par les brebis ayant agnelé durant les périodes de référence (la session de reproduction ou l'année). La structure de la matrice (R1) permet également d'approcher la fertilité du troupeau, à partir de l'évolution de l'effectif de cinq groupes de brebis :

$A=$ les brebis ayant agnelé au cours de la session de mise-bas étudiée;

$\mathrm{B}=$ les brebis n'ayant pas agnelé lors de la session, bien que réputées présentes lors de cette session et de la session de lutte correspondante.

Parmi celles-ci :

$\mathrm{G}=$ les brebis dont on sait qu'elles étaient en gestation au début de la session de lutte correspondante, c'est-à-dire qui ont agnelé dans les 150 jours précédant le début de la session de misebas étudiée ;

$J=$ les agnelles réputées trop jeunes pour être fertiles lors de la lutte, c'est-à-dire, dans notre situation, celles qui sont nées au cours de l'année civile de la session de mise-bas étudiée ou dans les 15 derniers jours de l'année précédente ;

$\mathrm{I}=$ les brebis qui ont mis bas moins de 40 jours avant la fin de la session de lutte, et que nous avons également considérées, après examen du fichier, comme non susceptibles d'être cyclée lors de cette lutte.

Sous ces conventions, l'estimateur retenu pour la fertilité s'écrit :

$$
\mathrm{f}=\mathrm{A} / \mathrm{A}+\mathrm{B}-(\mathrm{G}+\mathrm{J}+\mathrm{I})
$$

Ce calcul ne tient pas compte des avortements. Selon le cas, ceux-ci conduisent ou bien à sousestimer le numérateur (brebis poursuivant leur carrières de reproduction après l'épisode abortif) ou bien à sous-estimer à la fois le numérateur et le dénominateur (brebis n'agnelant plus par la suite,
En se fixant quelques règles, les informations du carnet d'agnelage permettent d'analyser les performances $d u$ troupeau au cours d'une période donnée et les performances de chaque brebis au cours de sa carrière.

Tableau 1. Analyse des résultats de reproduction du troupeau : structure de la matrice (R1).

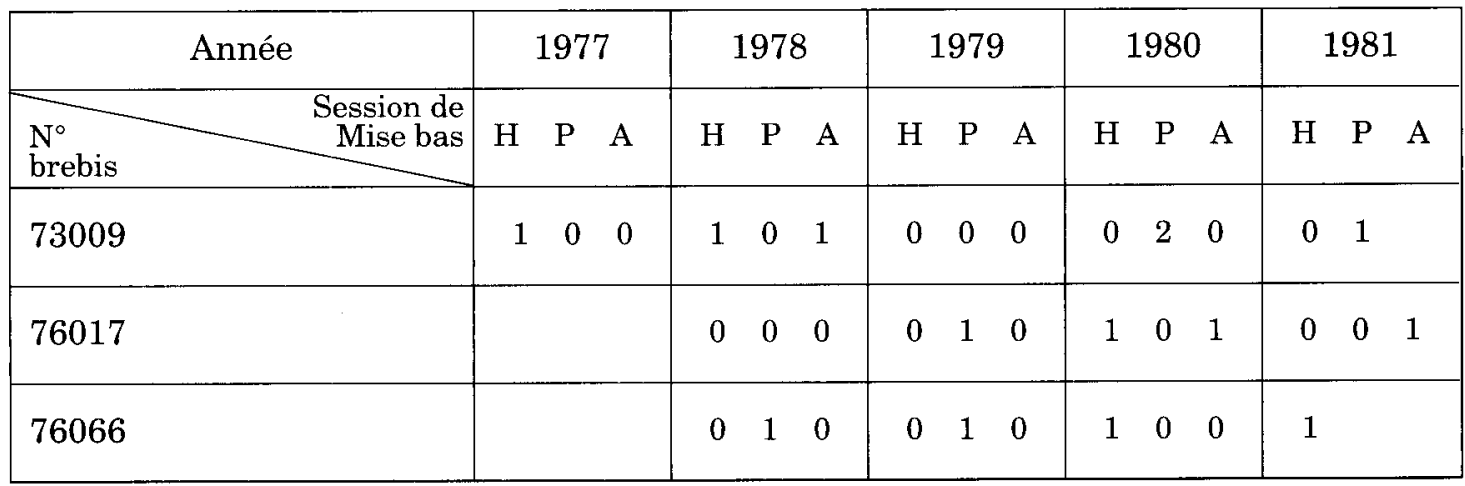

Brebis $n^{\circ} 73009$ : brebis née en 1973, entrée en reproduction avant le début de l'étude, réputée morte ou réformée à la suite de son agnelage de mars 1981

Brebis $n^{\circ} 76017$ : brebis ayant agnelé pour la première fois en mars 1979, à l'âge de 26 mois. Carrière se poursuivant audelà du tableau présenté

Brebis $n^{\circ} 76066$ : brebis ayant agnelé pour la première fois en mars 1978, à l'âge de 14 mois. Réputée sortie du troupeau à la suite de son agnelage de décembre 1980 . Sa carrière de reproduction est totalement couverte par la figure (exemple de "carrière complète"). 
et donc réputées à tort sorties du troupeau). Dans tous les cas, ceci débouche sur une légère sousévaluation de la fertilité réelle. Il est à noter qu'aucun épisode de maladie contagieuse abortive n'a été enregistré dans le troupeau durant la période d'étude. Par ailleurs, la convention adoptée pour la fixation de la date de sortie des brebis conduit à sous-estimer l'effectif des brebis présentées à la lutte et donc à surestimer la fertilité. L'intérêt de cette évaluation est enfin limité par l'impossibilité de distinguer, compte tenu de l'information disponible, les agneaux morts-nés, non viables et viables. Ceci nous fournit néanmoins un indicateur reproductible de la fertilité manifestée par le troupeau lors de chaque session

\section{Conventions de calcul}

Sur le plan méthodologique, la principale difficulté provient du fait que l'on ne dispose pas d'inventaires du troupeau régulièrement remis à jour, en sorte que l'on ne sait pas avec certitude si une brebis donnée était ou non présente lors d'une session de mise-bas donnée, si la case correspondante ne signale pas une mise-bas. Il était done nécessaire d'adopter des règles de décision à cet égard. Après une première étude des données, et divers entretiens avec l'éleveur, nous avons retenu les règles suivantes:

une brebis est réputée présente à toutes les sessions encadrées par deux mises-bas, sauf si l'écart excède 5 sessions de mise-bas consécutives, auquel cas il y a élimination de l'enregistrement.

- l'entrée des agnelles dans le troupeau de reproduction est réputée avoir lieu lors de la lutte d'été qui précède leur premier anniversaire, e'est-à-dire qu'elles apparaissent toujours pour la première fois (sous un indice de valeur égale, selon le cas, à 0,1 ou 2) dans une colonne correspondant à une mise-bas de décembre-janvier, à l'âge de un an.

- une brebis est réputée sortie du troupeau (morte ou réformée) lorsqu'elle n'apparaît plus dans les carnets d'agnelage durant au moins six périodes de mise-bas consécutives, soit deux années (durée fixée de manière empirique : certaines brebis ont présenté, dans l'exploitation étudiée des infertilités temporaires se traduisant par un "saut" maximum de cinq périodes consécutives). La date de sortie est conventionnellement fixée à la date de la dernière mise-bas connue.

Ces règles simples permettent de borner la période durant laquelle chaque brebis est réputée avoir été présente dans le troupeau. Seules sont considérées comme renseignées les cases de la matrice correspondant à des brebis réputées présentes, et le calcul de la période de présence de chaque brebís est donc suivi de l'effacement de tous les 0 contenus dans des cases extérieures à cette période. A la suite de cette opération, il est possible de distinguer, pour une période de mise-bas donnée, les brebis réputées présentes, bien que n'ayant pas agnelé (chiffre 0 dans la case correspondante), des brebis réputées absentes (case vide).

Un fichier complémentaire permet par ailleurs de relier chaque mise-bas, identifiée par le numéro de la brebis concernée et la session de mise-bas (saison, année), à sa date exacte (jour, mois, année). de reproduction, et nous permet d'évaluer la fécondité :

\section{$F=f \times \pi$}

Les données ainsi calculées ont été utilisées pour analyser les variations intra et interannuelles de ces paramètres. Nous avons en outre étudié la fertilité sous un aspect dynamique à partir des courbes résumant le "déroulement" des sessions de mise-bas, c'est-à-dire l'évolution de la fréquence cumulée des mises-bas au cours du temps, à l'intérieur d'une session de reproduction donnée. Ces courbes, d'allure générale comparable (sigmoïdes), présentent cependant une grande diversité, dont nous avons tenté de rendre compte simplement en classant les sessions selon le nombre de jours nécessaires pour atteindre $80 \%$ des mises-bas. Trois classes ont été retenues après examen de l'ensemble des données (moins de 21 jours, 21 à 41 jours, plus de 41 jours).

\section{b / Analyse des carrières individuelles}

Cette analyse vise à caractériser la succession des évènements de reproduction au cours de la carrière des brebis. Dans notre fichier, certaines carrières de reproduction sont complètement couvertes, mais beaucoup d'autres sont tronquées, soit qu'elles aient débuté avant le début de la période d'observation, soit qu'elles se soient prolongées au-delà de la fin de cette période. L'analyse n'a été conduite que sur des carrières complètes d'animaux nés entre 1976 et 1979 , de manière à éliminer le biais d'échantillonnage dû à la sélection des carrières courtes (Coulon et al 1990). La période d'observation (13 années) n'étant pas très longue par rapport à la longévité moyenne des animaux dans le système étudié, ceci limite considérablement l'échantillon, d'autant que la règle de décision adoptée pour fixer rétrospectivement les dates de sortie contribue à cette limitation. Au total, seules 114 carrières complètes, comptant 900 mises-bas au total, ont été conservées pour cette analyse.

La matrice (R2) utilisée pour ce travail dérive de la matrice (R1) par sélection des carrières complètes et par translation horizontale des lignes, aboutissant à caler à gauche la première case renseignée de chaque ligne. La colonne de gauche représente ainsi la première session de mise-bas suivant l'entrée en reproduction de chaque brebis (c'est-à-dire la première session hivernale suivant sa naissance). Dans le système étudié, toutes les agnelles de renouvellement sont issues de luttes d'été, et sont réputées entrer en reproduction au même âge. Chaque colonne regroupe donc des évènements survenus à des saisons comparables (mais pas nécessairement la même année), et l'âge peut facilement être repéré dans la structure de la matrice (R2), comme l'indique le tableau 2. A partir de ce tableau, les carrières de reproduction des brebis, symbolisées par une succession de 0,1 et 2, sont décrites de manière synthétique par les variables définies dans le tableau 3 .

Nous avons étudié dans un premier temps les valeurs moyennes et la dispersion de ces variables, ainsi que les relations entre variables prises deux à deux, par l'analyse de tableaux croi- 


\begin{tabular}{|c|c|c|c|c|c|c|c|c|c|c|c|c|c|c|c|}
\hline (Age) Année & \multicolumn{3}{|c|}{1} & \multicolumn{3}{|c|}{2} & \multicolumn{3}{|c|}{3} & \multicolumn{3}{|c|}{4} & \multicolumn{3}{|c|}{5} \\
\hline $\begin{array}{c}\mathrm{N}^{\circ} \text { session de } \\
\text { mise bas }\end{array}$ & 1 & 2 & & 4 & 5 & 6 & 7 & 8 & 9 & 10 & 11 & 12 & 13 & 14 & 15 \\
\hline $\mathrm{N}^{\circ}$ brebis $\quad$ Saison & $\mathrm{H}$ & $\mathrm{P}$ & A & $\mathrm{H}$ & $\mathrm{P}$ & A & $\mathrm{H}$ & $\mathrm{P}$ & $A$ & $\mathrm{H}$ & $\mathrm{P}$ & A & & & $\mathrm{A}$ \\
\hline 76017 & 0 & 0 & 0 & 0 & 1 & 0 & 1 & 0 & 1 & 0 & 0 & 1 & 0 & 0 & 1 \\
\hline 76066 & 0 & 1 & 0 & 0 & 1 & 0 & 1 & 0 & 0 & 1 & & & & & \\
\hline 83030 & 1 & 0 & 0 & 1 & 0 & 1 & 0 & 1 & 0 & 1 & 0 & 1 & 0 & 1 & 0 \\
\hline
\end{tabular}

Tableau 2. Analyse des carrières individuelles: structure de la matrice (R2).

Brebis $n^{\circ} 76017$ et 76066 : voir tableau 1 (translation horizontale des lignes)

Brebis $n^{\circ} 83030$ : $\quad$ brebis agnelant pour la première fois à l'âge de un an et maintenant par la suite en début de carrière un rythme régulier de trois mises bas tous les deux ans.

\begin{tabular}{|c|c|c|c|}
\hline Nom & Définition des variables & $\underset{\text { (1) }}{\text { Modalités }}$ & $\begin{array}{c}\text { Effectifs } \\
\text { (1) }\end{array}$ \\
\hline MB1 & $\begin{array}{l}\text { Age révolu à la première mise bas (en années) } \\
\text { valeurs entières égales à } 1,2 \text { ou } 3\end{array}$ & $\begin{array}{l}\mathrm{MB} 11=1 \\
\mathrm{MB} 12>1\end{array}$ & $\begin{array}{l}45 \\
69\end{array}$ \\
\hline SAISMB1 & Saison de la première mise bas & $\begin{array}{l}\text { SAISMB11 hiver } \\
\text { SAISMB12 printemps } \\
\text { SAISMB13 automne }\end{array}$ & $\begin{array}{l}63 \\
41 \\
10\end{array}$ \\
\hline MBTER & $\begin{array}{l}\text { Age révolu à la dernière mise bas (en années) } \\
\text { valeurs entières de } 1 \text { à } 10\end{array}$ & & \\
\hline VPROD & $\begin{array}{l}\text { Durée de la vie productive, de la première } \\
\text { fécondation à la dernière mise bas } \\
\text { (en années) } \\
\text { VPROD = MBTER - MB } 1+1 \\
\text { valeurs entières de } 1 \text { à } 10\end{array}$ & $\begin{array}{l}\text { VPROD1 } \leq 5 \\
\text { VPROD2 }=6 \\
\text { VPROD3 }=7 \text { ou } 8 \\
\text { VPROD }=9 \\
\text { VPROD5 }=10\end{array}$ & $\begin{array}{l}26 \\
13 \\
11 \\
21 \\
13\end{array}$ \\
\hline $\mathrm{NMB}$ & $\begin{array}{l}\text { Nombre total de mises bas } \\
\text { valeurs entières de } 1 \text { à } 13\end{array}$ & & \\
\hline NNES & $\begin{array}{l}\text { Nombre total d'agneaux nés } \\
\text { valeurs entières de } 1 \text { à } 14\end{array}$ & $\begin{array}{l}\text { NNES } 1<7 \\
\text { NNES } 2=7 \text { ou } 8 \\
\text { NNES3 }=9 \text { ou } 10 \\
\text { NNES } 4>10\end{array}$ & $\begin{array}{l}30 \\
26 \\
34 \\
24\end{array}$ \\
\hline PROL & $\begin{array}{l}\text { Prolificité (sur l'ensemble de la carrière) } \\
\text { présence de mises(s) bas double(s) }\end{array}$ & $\begin{array}{l}\text { PROL1 non } \\
\text { PROL2 oui }\end{array}$ & $\begin{array}{l}89 \\
25\end{array}$ \\
\hline NNESAN & $\begin{array}{l}\text { Productivité moyenne annuelle } \\
\text { (nombre moyen d'agneaux nés par an) } \\
\text { NNESAN = NNES/VPROD } \\
\text { valeurs continues de } 0,5 \text { à } 1,8\end{array}$ & $\begin{array}{l}\text { NNESAN1 }<1,0 \\
\text { NNESAN2 }=1 ; 1,1 \\
\text { NNESAN3 }=1,15 ; 1,3 \\
\text { NNESAN4 }>1,3\end{array}$ & $\begin{array}{l}16 \\
39 \\
39 \\
20\end{array}$ \\
\hline SAIS & $\begin{array}{l}\text { Indice de saisonnement : proportion des } \\
\text { mises bas ayant eu lieu en automne }\end{array}$ & $\begin{array}{l}\text { SAIS1 }<33 \% \\
\text { SAIS } 2 \geq 33 \%\end{array}$ & $\begin{array}{l}64 \\
50\end{array}$ \\
\hline FERT & $\begin{array}{l}\text { Indice de fertilité : proportion des mises bas } \\
\text { intervenant dans les } 21 \text { premiers jours de la } \\
\text { période de mise bas considérée }\end{array}$ & $\begin{array}{l}\text { FERT1 }>33 \% \\
\text { FERT } 2 \leq 33 \%\end{array}$ & $\begin{array}{l}49 \\
65\end{array}$ \\
\hline NOO & $\begin{array}{l}\text { Nombre de sauts de deux périodes de mise } \\
\text { bas consécutives } \\
\text { valeurs entières de } 0 \text { à } 6\end{array}$ & $\begin{array}{l}\text { NOO1 }=0 \\
\text { NOO2 }=1 \\
\text { NOO3 }=2 \\
\text { NOO4 }=3 \\
\text { NOO5 }>3\end{array}$ & $\begin{array}{l}15 \\
36 \\
23 \\
28 \\
13\end{array}$ \\
\hline NOOAN & $\begin{array}{l}\text { Nombre moyen de sauts de deux périodes } \\
\text { de mise bas par an } \\
\text { NOOAN = NOO/VPROD } \\
\text { valeurs continues de } 0 \text { à } 0,83\end{array}$ & $\begin{array}{l}\text { NOOAN1: }[0 ; 0,1[ \\
\text { NOOAN2 : }[01 ; 0,2[ \\
\text { NOOAN3 : }[0,2 ; 0,3[ \\
\text { NOOAN4 }:[0,3 ; 0,4[ \\
\text { NOOAN5 } \geq 0,4\end{array}$ & $\begin{array}{l}15 \\
24 \\
24 \\
26 \\
25\end{array}$ \\
\hline NOOO & $\begin{array}{l}\text { Nombre de sauts de trois périodes de mises } \\
\text { bas consécutives ou davantage } \\
\text { valeurs entières de } 0 \text { à } 3\end{array}$ & $\begin{array}{l}\text { NOOO1 }=0 \\
\text { NOOO } 2>0\end{array}$ & $\begin{array}{l}52 \\
62\end{array}$ \\
\hline
\end{tabular}

(1) Pour les variables retenues dans l'analyse factorielle des correspondances.

Tableau 3. Critères de description des carrières de reproduction des brebis. 
Tableau 4. Analyse combinée - Structure de la matrice (R3).

\section{La fertilité à la lutte de printemps est très variable mais ces variations sont largement tamponnées par le troupeau : le résultat annuel est beaucoup plus stable.}

\begin{tabular}{|c|c|c|c|c|c|c|c|c|c|c|c|c|}
\hline \multirow{2}{*}{\multicolumn{2}{|c|}{ 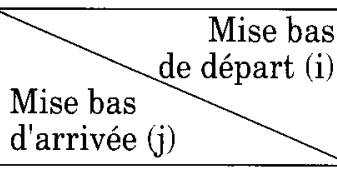 }} & \multicolumn{3}{|c|}{1982} & \multicolumn{3}{|c|}{1983} & \multicolumn{3}{|c|}{1984} & \multirow{2}{*}{$\begin{array}{l}\text { Primi } \\
\text { pares }\end{array}$} & \multirow{2}{*}{ Total } \\
\hline & & $\mathrm{P}$ & A & $\mathrm{H}$ & $\mathrm{P}$ & A & $\mathrm{H}$ & $\mathrm{P}$ & A & $\mathrm{H}$ & & \\
\hline 1983 & $\begin{array}{l}\mathrm{H} \\
\mathrm{P}\end{array}$ & $\begin{array}{c}42 \\
7\end{array}$ & $\begin{array}{c}0 \\
42\end{array}$ & 0 & & & & F & & & $\begin{array}{r}28 \\
8\end{array}$ & $\begin{array}{l}90 \\
61\end{array}$ \\
\hline & A & 1 & 15 & 13 & 2 & & & & & & 8 & 40 \\
\hline 1984 & $\begin{array}{l}\mathrm{H} \\
\mathrm{P} \\
\mathrm{A}\end{array}$ & 1 & $\begin{array}{l}7 \\
2\end{array}$ & $\begin{array}{c}40 \\
10 \\
4\end{array}$ & $\begin{array}{c}42 \\
8 \\
1\end{array}$ & $\begin{array}{c}0 \\
19 \\
17\end{array}$ & $\begin{array}{c}0 \\
44\end{array}$ & 16 & & & $\begin{array}{r}12 \\
5 \\
9\end{array}$ & $\begin{array}{r}102 \\
44 \\
91\end{array}$ \\
\hline & $\mathrm{H}$ & & & & 1 & 2 & 26 & 13 & 2 & & 16 & 60 \\
\hline 1985 & $\begin{array}{l}\mathrm{P} \\
\mathrm{A}\end{array}$ & & & & & 0 & $\begin{array}{c}15 \\
3\end{array}$ & $\begin{array}{l}4 \\
1\end{array}$ & $\begin{array}{l}42 \\
25\end{array}$ & $\begin{array}{c}0 \\
26\end{array}$ & $\begin{array}{r}24 \\
5\end{array}$ & $\begin{array}{l}85 \\
61\end{array}$ \\
\hline Mort et Réforme & & 10 & 17 & 23 & 7 & 2 & 14 & 9 & 9 & 8 & & \\
\hline TOTAL & & 65 & 83 & 90 & 61 & 40 & 102 & 44 & 91 & 60 & & \\
\hline
\end{tabular}

sés pour les variables discrètes, et l'étude des corrélations pour celles d'entre elles qui peuvent être assimilées à des variables continues. Nous avons ensuite réalisé une analyse factorielle des correspondances (AFC) suivie d'une classification automatique par la méthode des nuées dynamiques, en vue d'établir une typologie des carrières de reproduction des brebis. Toutes ces analyses ont utilisé les programmes du progiciel LEAS (Escoufier et al 1987).

\section{c / Analyse combinée}

Nous désignons sous ce terme l'ensemble des méthodes qui combinent des analyses menées à l'échelle du troupeau et à celle des individus. Nous en fournissons deux exemples :

Figure 1. Profils de fertilité par campagne et fertilité moyenne annuelle (PA : profils atypiques).

Les données postérieures à 1987 n'ont pu être utilisées pour cette figure compte tenu du recul nécessaire pour évaluer l'effectif des brebis présentes à chaque session de reproduction.

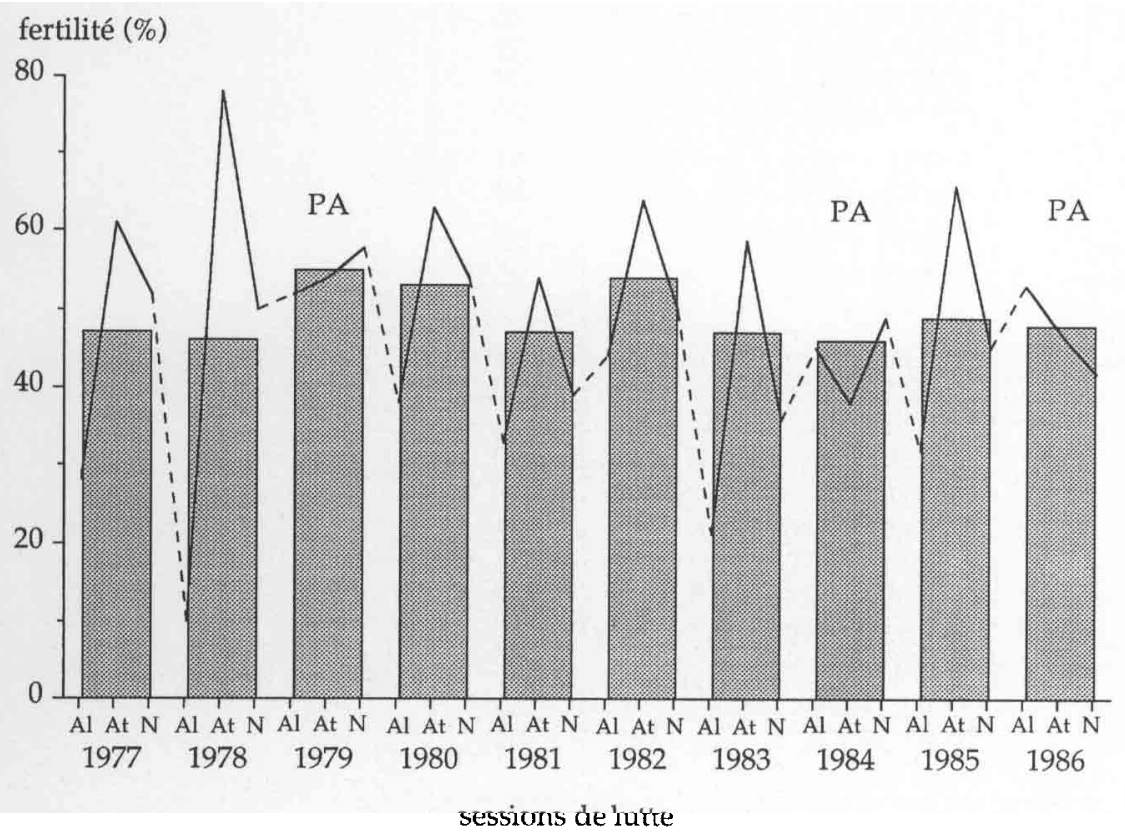

- l'analyse des rythmes de reproduction a été menée sur une matrice (R3) constituée à partir de l'examen des carrières individuelles des brebis, et qui résume l'information relative aux "reports" d'une session de mise-bas à une autre (tableau 4). Dans chaque case ( $\mathrm{i} \times \mathrm{j}$ ) de cette matrice figure l'effectif des brebis qui, ayant agnelé lors de la session de mise-bas i (mise-bas "de départ"), ont réalisé leur mise-bas suivante lors de la session $j$ (mise-bas "d'arrivée"). Pour l'ensemble des brebis ayant mis bas au cours d'une session donnée, il est ainsi possible de retrouver la date des sessions durant lesquelles a eu lieu la mise-bas précédente (lecture horizontale) ou la mise-bas suivante (lecture verticale). Les différences entre les totaux marginaux (nombre total de mises-bas enregistrées pour la session considérée) et le cumul des effectifs des cases des lignes ou des colonnes correspondent respectivement aux effectifs des agnelles ayant mis bas pour la première fois lors de la session considérée ou à ceux des brebis sorties du troupeau à la suite de cette session.

- l'analyse des interactions entre les comportements individuels des animaux et les variations du milieu d'élevage a été conduite en étudiant, selon leur type de carrière, les réactions des brebis face à différentes perturbations repérées par les variations des performances de fertilité du troupeau.

\section{2 / Résultats}

\section{1 / Analyse des performances à l'échelle du troupeau}

\section{a / Fertilité}

La figure 1 présente les valeurs estimées de la fertilité pour l'ensemble des sessions de reproduction de la période étudiée. La saison est le principal facteur de variation de la fertilité, qui s'établit en moyenne à 0,36 pour la session de lutte d'avril (contre-saison), 0,60 pour la session d'août et 0,50 pour la session de novembre (en pleine saison sexuelle). La fertilité moyenne, calculée sur une 
base annuelle à partir de trois sessions de reproduction consécutives, s'établit à 0,49 . Les coefficients de variation de la fertilité, très élevés pour la lutte d'avril (39\%), encore élevés pour la session de lutte d'août (18\%), sont beaucoup plus faibles pour la session de novembre (12\%) et pour la moyenne annuelle $(7 \%)$. La lutte d'avril donne donc, en termes de fertilité, des résultats extrêmement variables dans le système considéré. Cependant, ces variations sont largement tamponnées par le troupeau, en sorte que le résultat annuel est beaucoup plus stable que celui qui est enregistré pour chacune des sessions, et particulièrement pour la lutte d'avril : il existe donc à ce niveau des phénomènes de compensation.

Les contributions respectives des différentes sessions de reproduction à la fertilité moyenne annuelle permettent de définir le "profil de fertilité" d'une année donnée. Dans 7 cas sur 10, ces profils se rattachent au type moyen : fertilité minimum à la lutte d'avril, maximum en août, valeur intermédiaire en novembre (figure 1). Trois campagnes se distinguent par rapport à ce modèle : $1979-1980 ; 1984-1985 ; 1986-1987$. C'est au cours de ces campagnes qu'ont été enregistrées les meilleures performances de fertilité à la lutte d'avril. La réussite de cette session de lutte apparaît ainsi comme le principal facteur de variation des profils de fertilité annuels. L'analyse de ces profils révèle également que les trois campagnes caractérisées par des profils atypiques ont succédé aux campagnes marquées par les plus faibles fertilités à la lutte d'avril, et les plus forts écarts de fertilité entre sessions de lutte d'avril et d'août d'une part, d'août et de novembre d'autre part. L'existence de phénomènes de régulation à la fois intra et inter-annuelle de la fertilité est confirmée par la corrélation négative qui est observée entre la fertilité de la session de lutte d'avril et celle de la lutte d'août qui lui fait suite $(\mathrm{r}=-0,71, \mathrm{p}<$ 0,05 ). Autrement dit, la fertilité à la lutte d'avril explique la moitié de la variance de la fertilité à la lutte d'août. La fertilité à la session de lutte de novembre, peu variable, n'est pour sa part pas significativement corrélée à celle des sessions d'août ou d'avril qui précèdent. L'origine des phénomènes ainsi mis en évidence est à rechercher au niveau des rythmes de reproduction individuels des animaux.

\section{b / Prolificité et fécondité}

La fréquence des mises-bas multiples est très faible, ce qui limite les analyses envisageables. La valeur moyenne de la prolificité, toutes sessions de reproduction et toutes années confondues est de 1,05 . La variabilité saisonnière est peu marquée : les valeurs moyennes pour les sessions de lutte d'avril d'août et de novembre sont respectivement de $1,02,1,05$ et 1,09 (différences non significatives). En termes de fécondité, les moyennes saisonnières s'élèvent respectivement à $0,37,0,63$ et 0,55 agneaux par brebis potentiellement fertile lors de la lutte.

La variabilité interannuelle de la prolificité est faible (écart-type $=0,02$ ). Aucune relation entre fertilité et prolificité n'a pu être mise en évidence.

\section{c / Analyse du déroulement des mises-bas}

Les courbes d'évolution de la fréquence cumulée des mises-bas au cours du temps présentent des allures différentes d'une session de reproduction à l'autre. Les exemples que nous avons retenus pour la figure 2 en illustrent les principaux types. La courbe relative à la mise-bas du printemps 1980 caractérise une session de reproduction sans problème particulier. La rupture de pente observée à la fin de la session reflète la physionomie de la lutte, et montre que des fécondations continuaient à se produire lorsque les béliers ont été retirés du troupeau, fin novembre 1979. L'allure de la misebas d'automne 1981 reflète des conditions de lutte moins favorables : fertilité réduite (lutte de contre-saison) et courbe irrégulière. Le plateau observé en début de session correspond très probablement au stress dù à la mise à l'herbe, qui s'est effectuée durant la lutte. Enfin, la physionomie de la mise-bas de l'automne 1983 témoigne des très mauvaises conditions dans lesquelles s'est déroulée la lutte d'avril cette année-là : faible fertilité des brebis au sortir de l'hiver et mauvaises conditions météorologiques à la mise à l'herbe, se traduisant par une courbe particulièrement heurtée. La prolongation de la durée de la session de lutte au-delà de 45 jours est restée pratiquement sans résultat.

Le critère synthétique retenu dans un premier temps (nombre de jours nécessaires à la réalisation de $80 \%$ des mises-bas) ne suffit pas à rendre compte de cette variabilité. Il convient donc d'uti-

Figure 2. Evolution du pourcentage cumulé de mises bas en fonction du temps écoulé depuis le début de la session de mise bas au cours de différentes sessions de reproduction (en \% de brebis réputées fertiles).

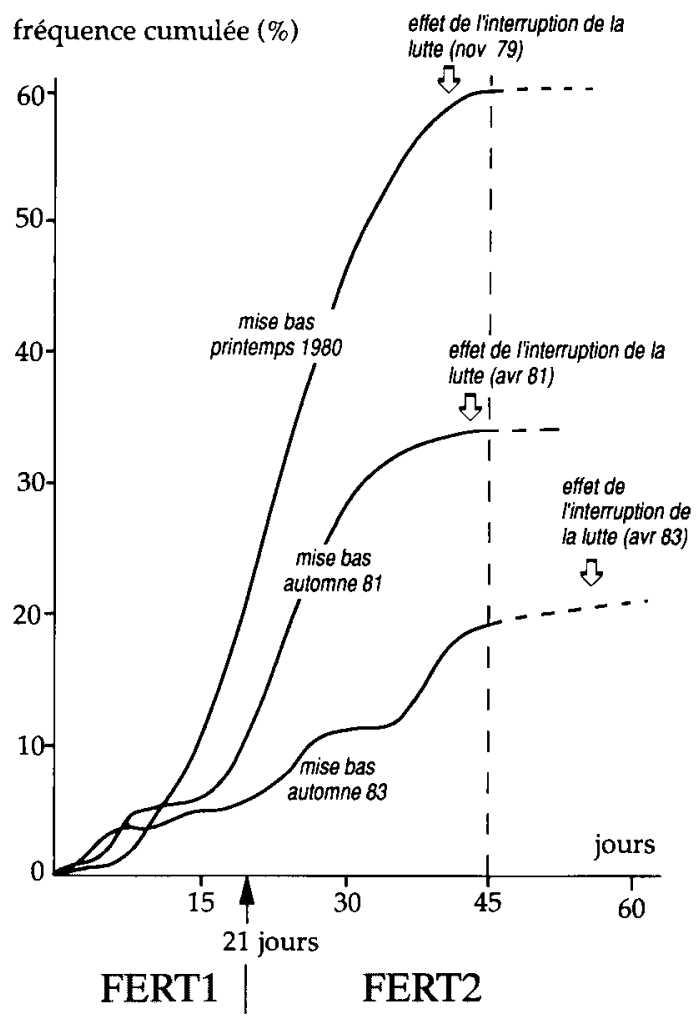


Tableau 5. Valeurs moyennes et écarts-types des principales variables utilisées pour décrire les carrières des brebis.

\begin{tabular}{|l|c|c|}
\hline \multicolumn{1}{|c|}{ Variable } & Moyenne & Ecart-type \\
\hline MBTER âge à la dernière mise bas (années) & 7,6 & 2,22 \\
VPROD duré de vie productive (années) & 7,0 & 2,33 \\
NMB & 7,9 & 2,89 \\
NNEmbre de mises bas & 8,2 & 3,07 \\
NNESAN productivité mogneaux nés & 1,2 & 0,22 \\
NOO nomnue annelle & 2,0 & 1,39 \\
NOOAN & 0,27 & 0,18 \\
NOOO noyenne annuelle deux sauts de deux périodes & 0,88 & 0,93 \\
\hline
\end{tabular}

liser simultanément plusieurs indicateurs de ce type. Nous n'avons cependant pas approfondi cette analyse, compte tenu de la difficulté d'interpréter la variabilité ainsi mise en évidence, faute de données relatives aux conditions dans lesquelles se sont déroulées les sessions de lutte (état des brebis à la mise en lutte, alimentation, pratiques d'élevage, etc). Seuls, des suivis d'élevages permettraient de dégager les clés d'interprétation qui font actuellement défaut. Pour l'étude des carrières individuelles de reproduction (voir ci-dessous), nous avons préféré retenir un indicateur (FERT) défini comme la proportion des mises-bas intervenant dans les 21 premiers jours de la session de mise-bas considéré (tableau 3 ). Ces misesbas sont le fait de brebis qui peuvent être considérées comme étant cyclées lors de la mise en lutte, le nombre de 21 jours correspondant à la durée moyenne des cycles - 17 jours- augmentée de deux fois la valeur de l'écart-type de la durée de gestation en race Préalpes (Ricordeau et Flamant 1969).

Figure 3. Distribution de

l'âge à la dernière mise bas (MBTER) dans l'échantillon étudié.

fréquence (\%)

$(n=114)$

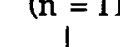

20
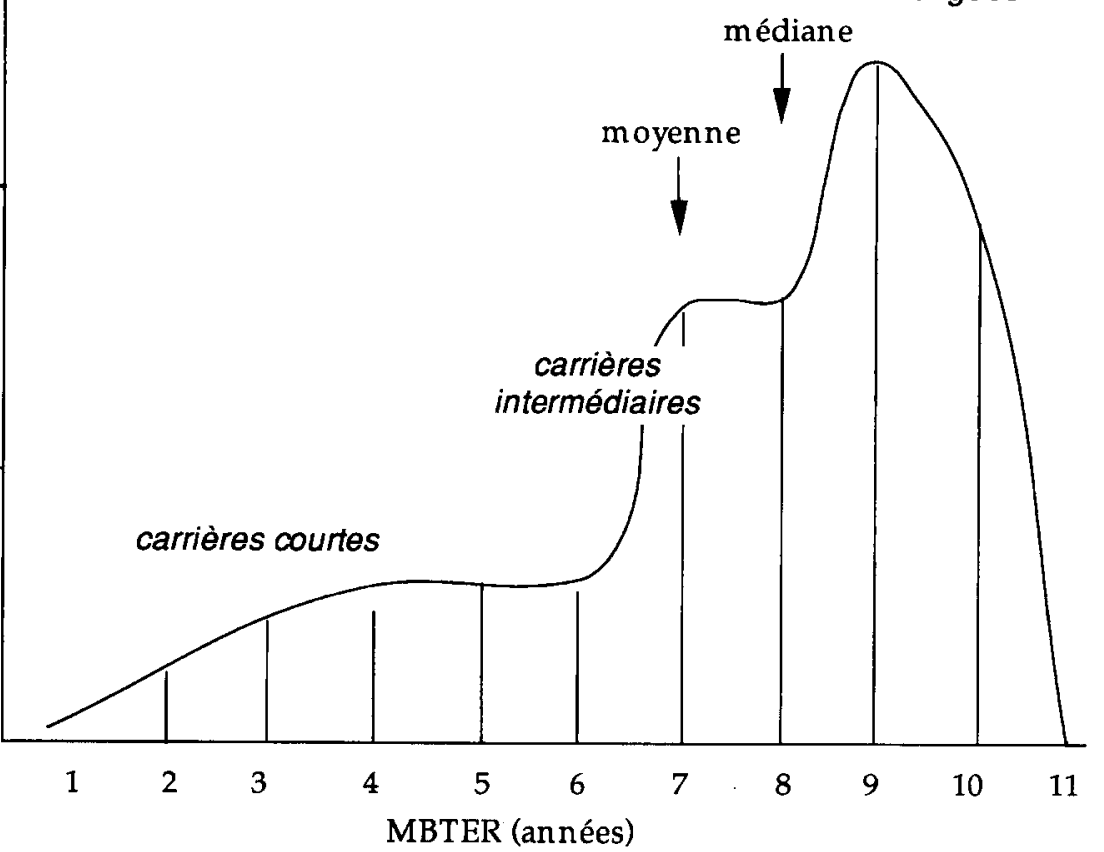

\section{2 / Analyse des carrières}

\section{a / Analyse descriptive des variables et de leurs relations deux à deux}

Les valeurs moyennes et les écarts-types des principales variables utilisées pour décrire les carrières des brebis sont présentées dans le tableau 5 .

La variabilité apparaît très forte pour l'ensemble des critères étudiés. Seul, le nombre d'agneaux nés par année de vie productive (NNESAN) présente un cœfficient de variation inférieur à $20 \%$. La variabilité est particulièrement élevée pour les critères relatifs aux "sauts" de plusieurs sessions de reproduction consécutives (N00, N00AN et N000), ceci reflétant à la fois la nature des données de base (caractères discrets prenant un nombre limité de valeurs) et la grande diversité des comportements individuels.

L'âge à la dernière mise-bas (MBTER) représente un bon indicateur de la longévité des brebis. Plus que sa valeur moyenne (qu'il serait intéressant de comparer entre élevages ou entre types génétiques), c'est la distribution de cette variable qui retiendra l'attention dans le cadre de cette étude (figure 3). Environ $45 \%$ des brebis effectuent dans le système considéré des carrières que l'on peut considérer comme longues, conduisant à une dernière mise-bas à l'âge de 9 ou 10 ans, ce qui est tout à fait remarquable puisque l'âge à la dernière mise-bas ne dépasse jamais 10 ans. C'est dire que près de la moitié des brebis sont réformées à un âge proche de cette limite, qui apparaît comme un seuil physiologique dans le système étudié.

Les autres brebis réalisent ou bien des carrières de durée intermédiaire, et mettent bas pour la dernière fois à l'âge de 7 ou 8 ans $(n=36)$, ou bien des carrières courtes, la dernière mise-bas intervenant entre 1 et 6 ans $(n=27)$.

L'âge à la dernière mise-bas s'analyse, selon nos conventions de calcul (qui entraînent une surestimation de la durée de vie productive), comme la somme de deux composantes : l'âge à la première mise-bas (MB1) et la durée de la vie productive (VPROD). Ces composantes sont-elles indépendantes? La comparaison des durées moyennes de la vie productive des brebis selon leur âge à la première mise-bas montre que les brebis ayant agnelé à l'âge de un an présentent une vie productive en 
moyenne plus longue que les autres ( 7,8 contre 6,4 années), le second groupe rassemblant des brebis ayant mis bas pour la première fois à 2 ans ( $93 \%$ des cas) ou à 3 ans (7\%). Les distributions de la durée de vie productive dans ces deux sous-populations diffèrent significativement $(\mathrm{p}<0,05)$. La figure 4 illustre ce résultat, et montre que ces distributions sont en réalité décalées d'environ un an sur l'axe des abcisses. Ce décalage correspond à la différence d'âge à l'entrée en reproduction. Si l'on fait glisser vers la gauche la courbe correspondant au groupe des brebis ayant mis bas à un an, de manière à corriger ce décalage initial, la similitude de l'allure des deux courbes apparaît de manière frappante. Ceci montre que l'âge exerce un effet propre sur le profil de carrière, et confirme que l'âge de 10 ans révolus représente bien, en termes de longévité, un butoir physiologique dans le système considéré (en d'autres termes, aucune brebis n'atteint son 11 è anniversaire) : la durée maximum de la vie productive est donc limitée par une entrée en reproduction tardive.

Figure 4. Distribution de la durée de vie productive selon l'âge à la première mise bas $(n=114)$

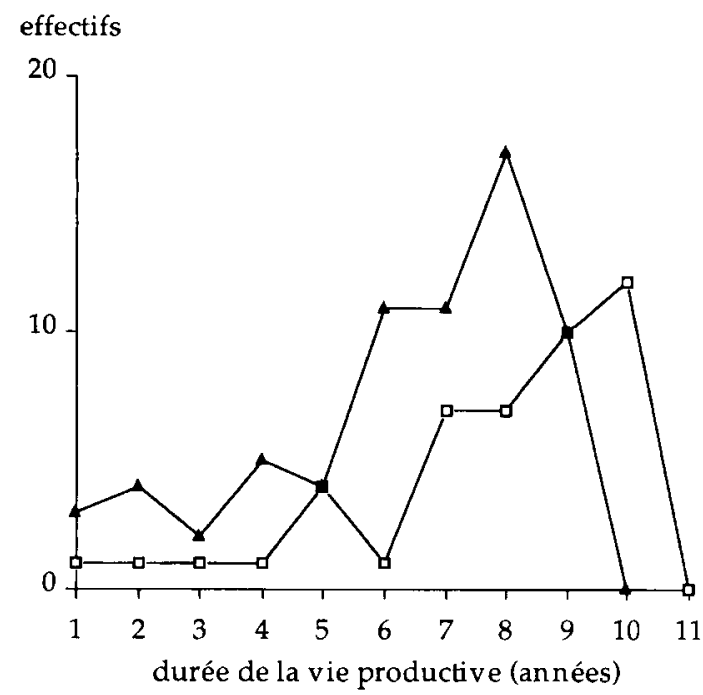

première mise bas

ㅁ à 1 an $(n=45)$

- à 2 ans ou plus $(n=69)$

Le nombre moyen de mises-bas par brebis s'élève à 7,95 soit en moyenne 1,14 agnelages par an, pour une production de 1,17 agneau né par an. En raison de son effet sur la durée de vie productive, l'âge à l'entrée en reproduction exerce un effet sensible sur ces variables. Les brebis agnelant pour la première fois à un an mettent bas en moyenne 8,71 fois dans leur carrière, contre 7,45 fois seulement pour les autres $(\mathrm{p}<0,05)$. Au total, les brebis de chacune de ces deux catégories donnent en moyenne respectivement naissance, sur l'ensemble de leur carrière, à 8,96 et 7,80 agneaux, soit un avantage de plus d'un agneau en faveur des brebis les plus précoces. Cependant, les brebis entrées tardivement en reproduction maintiennent en moyenne un rythme de reproduction légèrement supérieur (1,17 mise-bas par année de vie productive contre 1,10 pour les brebis ayant agnelé à un an) et manifestent une prolificité plus élevée $(1,047$ contre 1,028$)$, débouchant sur une productivité annuelle supérieure : 1,23 agneaux nés par an en moyenne contre $1,13(\mathrm{p}<0,05)$.

L'étude des corrélations entre variables n'apporte pas beaucoup d'information supplémentaire : l'âge à la dernière mise-bas, la durée de la vie productive, le nombre de mises-bas et le nombre total d'agneaux nés sont étroitement liés, comme on pouvait s'y attendre (cœfficients de corrélation tous supérieurs à 0,88 ). Le nombre moyen d'agneaux nés par an (NNESAN), qui est un indice de la productivité annuelle des brebis, apparaît positivement, mais faiblement lié aux variables $\mathrm{NMB}$ et NNES $(\mathrm{r}=0,3$ et 0,4 respectivement). Il n'est en revanche pas lié aux variables MBTER et VPROD, et constitue donc un indicateur de productivité indépendant de la durée des carrières.

Le nombre de sauts de deux sessions de reproduction consécutives (NOO), qui contribue à caractériser le rythme de reproduction, est positivement lié aux quatre variables qui expriment la longévité des brebis (MBTER, VPROD, NMB et NNES). La variable NOOAN (nombre moyen de sauts de deux périodes par année de vie productive), construite pour s'affranchir de cet effet, n'est liée qu'à la variable $\mathrm{NOO}(\mathrm{r}=0,86)$.

La variable NOOO (nombre de sauts de plus de deux périodes consécutives), conçue comme un indicateur des infertilités temporaires, ne pouvait être assimilée à une variable continue, car elle ne prend que quelques valeurs. Nous avons donc étudié ses relations éventuelles avec les autres variables à partir de tableaux croisés. Aucune liaison n'a pu être décelée avec les variables MB1 (âge à la première mise-bas), SAIS (indice de saisonnement) et FERT (indice de fertilité). C'est dire que les brebis entrées plus ou moins précocement en reproduction sont également affectées par l'infertilité temporaire, et que les caractères de saisonnement et de fertilité (cyclicité en début de lutte) ne sont pas non plus liés à l'apparition d'infertilités temporaires, dans le troupeau étudié. La probabilité de subir un ou plusieurs épisodes d'infertilité temporaire est d'autant plus élevée que la carrière est plus longue $(\mathrm{p}<0,05)$. Une liaison négative et plus étroite $(p<0,01)$ est décelée entre les variables NOOO et NNESAN : l'infertilité temporaire s'accompagne logiquement d'une baisse de la productivité. Il n'y a aucun lien décelable entre les variables NOOO et NOO, ce qui semble confirmer qu'il s'agit bien de phénomènes de nature différente. Les brebis "prolifiques" (celles qui ont mis bas au moins une portée double, soit 25 brebis sur 114 , pour 33 mises-bas gémellaires) présentent en moyenne moins d'épisodes d'infertilité que les autres sur l'ensemble de leur carrière (NOOO $=0,64$ contre 0,94 ), une liaison négative étant décelable entre les variables PROL et NOOO $(\mathrm{p}<0,05)$. Cette liaison n'est pas due à un biais provenant d'une différence de longévité. Bien au contraire, la durée moyenne de la vie productive s'établit à 7,9 années pour les brebis prolifiques contre 6,7 pour les autres $(\mathrm{p}<0,05)$.
La durée maximum de la vie productive est liée à l'âge à la première mise bas, l'âge à la dernière mise bas ne dépassant jamais 10 ans 


\section{b / Analyse multivariée et typologie des carrières de reproduction des brebis}

L'analyse des correspondances et la classification permettent d'avoir une vue plus synthétique sur ces relations, et de caractériser globalement les carrières des brebis.

Figure 5. Position des variables les plus discriminantes pour l'axe 1 (O) et l'axe 2 ( $\mathbf{A}$ ) de l'AFC et localisation des types de carrière issus de la classification automatique

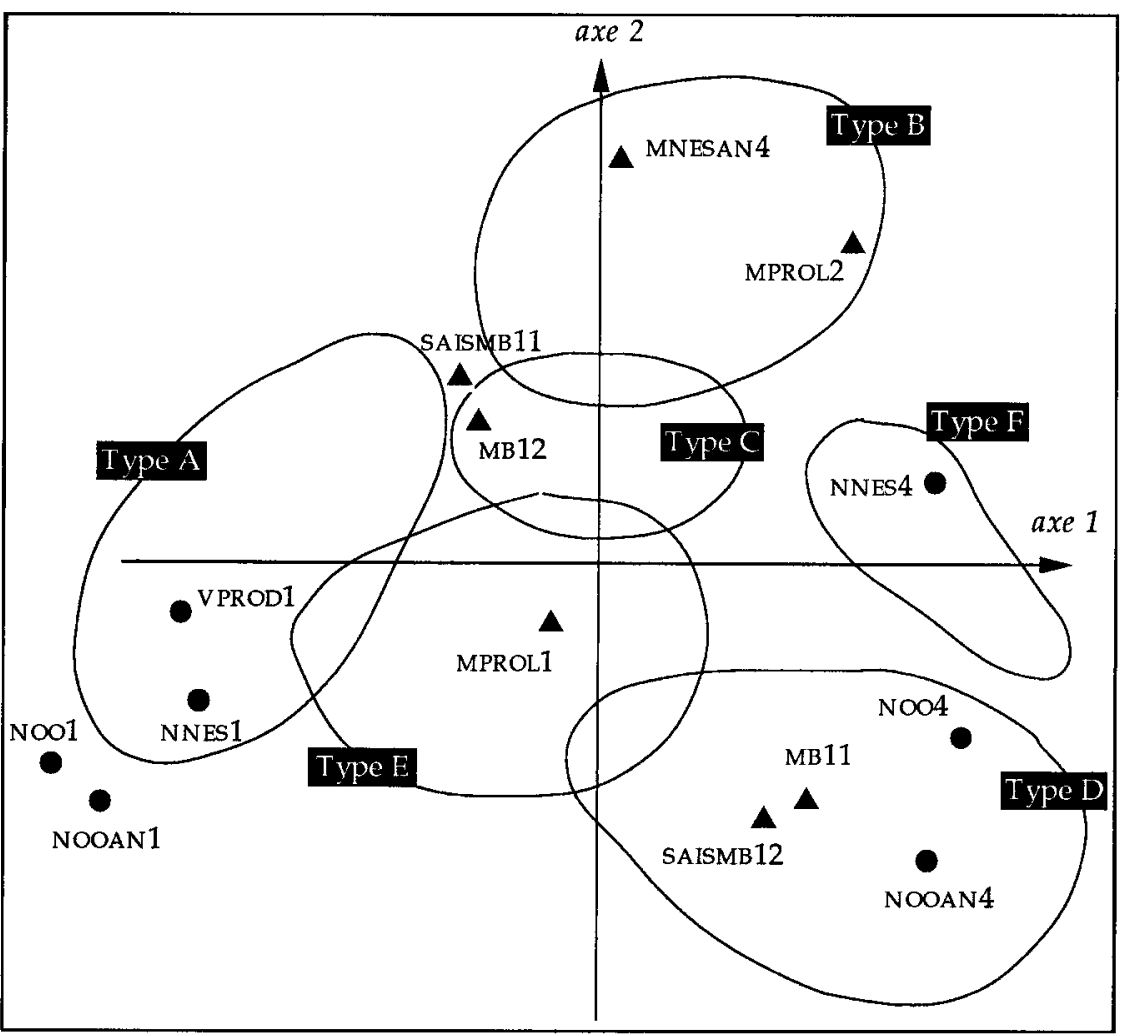

\section{Analyse factorielle des correspondances}

La part de la variance totale expliquée par les trois premiers axes de l'AFC s'élève respectivement à $12,7,9,9$ et $9,1 \%$, soit $31,7 \%$ au total. Nous nous bornerons à l'interprétation de ces trois premiers axes, en nous appuyant sur les figures 5 et 6 .

L'axe 1 oppose : à gauche, les modalités des variables liées à des carrières courtes (VPROD1, NNES1, NOO1) et à un rythme de reproduction très soutenu, de l'ordre de trois mises-bas tous les deux ans (NOOAN1) ; à droite, celles qui sont liées à des carrières longues (NNES4, N004) et à un rythme de reproduction annuel (N00AN4). Il s'interprète clairement en termes de longévité.

Sur l'axe 2, les variables les plus discriminantes sont : vers le haut une prolificité élevée (PROL2), une forte productivité moyenne annuelle (NNESAN4), des premières mises-bas tardives (MB12) et d'hiver (SAISMB11) ; vers le bas, des premières mises-bas précoces (MB11) et au printemps (SAISMB12). La liaison entre l'âge à la première mise-bas et la saison à laquelle elle survient est expliquée par la figure 7, qui décrit l'entrée en reproduction des agnelles dans le système étudié.

Sur l'axe 3 s'opposent : en bas un nombre élevé d'agneaux nés (NNES4) et l'absence d'infertilité temporaire (NOOO1) ; en haut la présence d'infertilités temporaires (NOOO2), un faible nombre de sauts de deux périodes de mise-bas consécutives (NOO2 et NOOAN2).

Les axes 2 et 3 peuvent tous deux s'interpréter en termes de productivité, l'axe 2 privilégiant les composantes prolificité et circonstances de la première mise-bas, l'axe 3 le rythme de reproduction sur l'ensemble de la carrière.

Figure 6. Evolution des modalités de différentes variables sur le plan factoriel $1 \times 2$. (les fortes valeurs des différentes variables correspondent aux modalités de rang élevé).

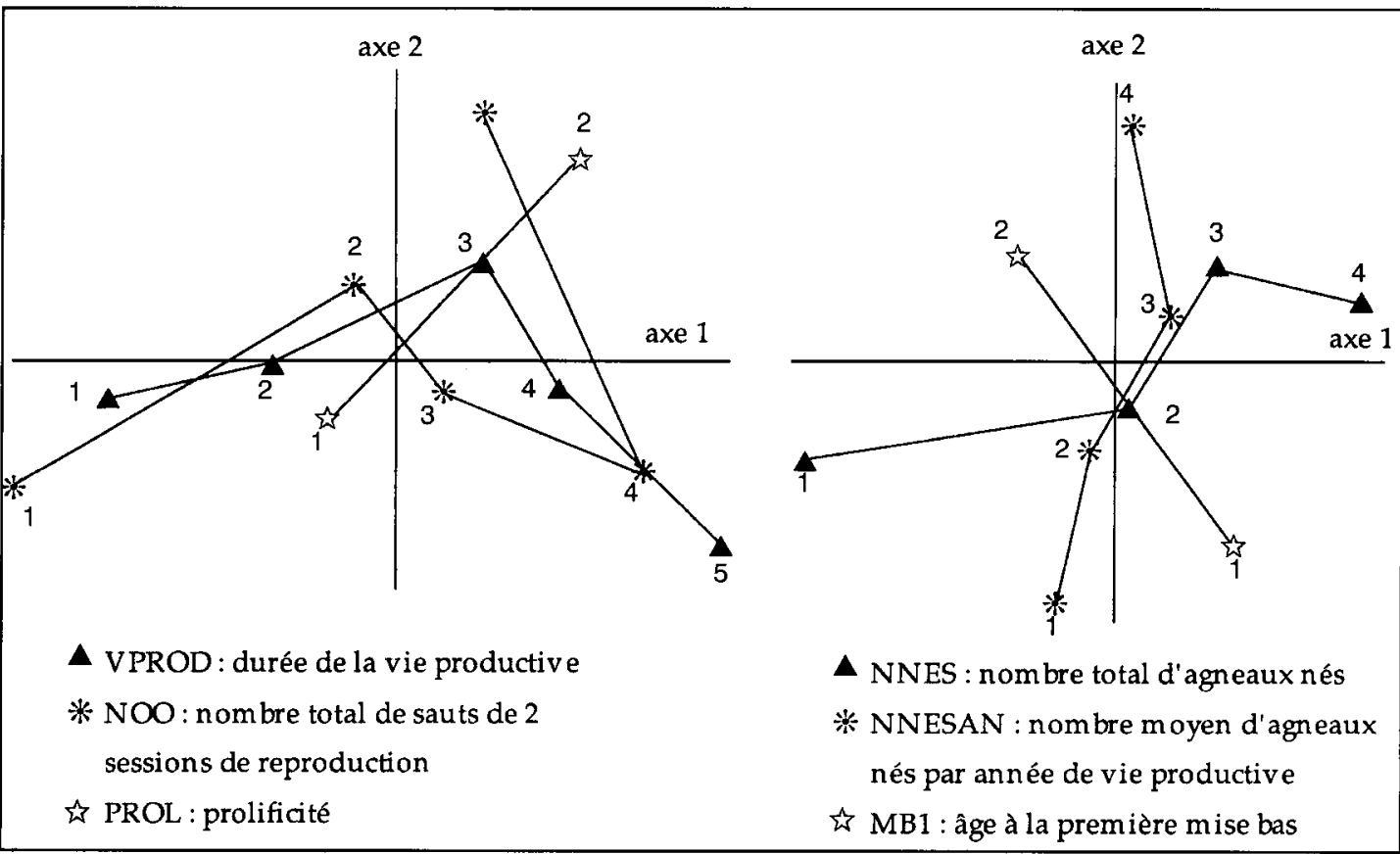


Tableau 6. Caractéristiques descriptives des différents types de carrières distingués par la classification automatique.

\begin{tabular}{|c|c|c|c|c|c|c|c|c|c|c|c|c|c|}
\hline \multirow{2}{*}{$\begin{array}{l}\text { Type de } \\
\text { carrière }\end{array}$} & \multirow{2}{*}{ Effectif } & \multicolumn{2}{|c|}{$\begin{array}{l}\text { Première } \\
\text { mise bas }\end{array}$} & \multirow[b]{2}{*}{ MBTER } & \multirow[b]{2}{*}{ NMB } & \multirow[b]{2}{*}{ PROL } & \multirow[b]{2}{*}{ NNES } & \multirow[b]{2}{*}{ NNESAN } & \multicolumn{2}{|c|}{$\begin{array}{l}\text { Rythme de } \\
\text { reproduction }\end{array}$} & \multicolumn{2}{|c|}{ Saisonnalité } & \multirow{2}{*}{$\begin{array}{c}\text { Fertilité } \\
\text { FERT }\end{array}$} \\
\hline & & MB1 & SAISMB1 & & & & & & NOOAN & N000 & DISTRIB & SAIS & \\
\hline A & 17 & $\begin{array}{c}2 \\
80 \%\end{array}$ & $\begin{array}{lr}\mathrm{A} & 0 \\
\mathrm{H} & 88 \\
\mathrm{P} & 12\end{array}$ & $\begin{array}{c}5,6 \\
(1,9)\end{array}$ & $\begin{array}{c}4,8 \\
(2,7)\end{array}$ & $\begin{array}{c}0 \\
100 \%\end{array}$ & $\begin{array}{c}4,8 \\
(2,7)\end{array}$ & $\begin{array}{c}1,28 \\
(0,26)\end{array}$ & $\begin{array}{c}0,22 \\
(0,25)\end{array}$ & $\begin{array}{c}0 \\
100 \%\end{array}$ & $\begin{array}{ll}\mathrm{A} & 37 \\
\mathrm{H} & 35 \\
\mathrm{P} & 28\end{array}$ & & $\begin{array}{c}1 \\
53 \%\end{array}$ \\
\hline B & 15 & $\begin{array}{c}2 \\
80 \%\end{array}$ & $\begin{array}{lr}\mathrm{A} & 7 \\
\mathrm{H} & 93 \\
\mathrm{P} & 0\end{array}$ & $\begin{array}{c}9,3 \\
(0,9)\end{array}$ & $\begin{array}{c}9,7 \\
(1,4)\end{array}$ & $\begin{array}{c}\geq 1 \\
75 \%\end{array}$ & $\begin{array}{c}10,6 \\
(1,35)\end{array}$ & $\begin{array}{c}1,34 \\
(0,15)\end{array}$ & $\begin{array}{c}0,40 \\
(0,23)\end{array}$ & $\begin{array}{c}0 \\
80 \%\end{array}$ & $\begin{array}{ll}\mathrm{A} & 41 \\
\mathrm{H} & 29 \\
\mathrm{P} & 30\end{array}$ & $\begin{array}{c}2 \\
73 \%\end{array}$ & $\begin{array}{c}2 \\
66 \%\end{array}$ \\
\hline $\mathrm{C}$ & 12 & $\begin{array}{c}2 \\
90 \%\end{array}$ & $\begin{array}{lr}\mathrm{A} & 8 \\
\mathrm{H} & 75 \\
\mathrm{P} & 17\end{array}$ & $\begin{array}{c}9,3 \\
(1,3)\end{array}$ & $\begin{array}{c}8,8 \\
(1,1)\end{array}$ & $\begin{array}{c}0 \\
100 \%\end{array}$ & $\begin{array}{c}8,8 \\
(1,1)\end{array}$ & $\begin{array}{c}1,13 \\
(0,09)\end{array}$ & $\begin{array}{c}0,14 \\
(0,04)\end{array}$ & $\begin{array}{c}\geq 1 \\
100 \%\end{array}$ & $\begin{array}{ll}\mathrm{A} & 38 \\
\mathrm{H} & 31 \\
\mathrm{P} & 31\end{array}$ & $\begin{array}{c}2 \\
66 \%\end{array}$ & $\begin{array}{c}2 \\
92 \%\end{array}$ \\
\hline D & 11 & $\begin{array}{c}1 \\
100 \%\end{array}$ & \begin{tabular}{|lr}
$\mathrm{A}$ & 0 \\
$\mathrm{H}$ & 0 \\
$\mathrm{P}$ & 100 \\
\end{tabular} & $\begin{array}{c}9,0 \\
(1,8)\end{array}$ & $\begin{array}{c}9,7 \\
(2,6)\end{array}$ & $\begin{array}{c}0 \\
82 \%\end{array}$ & $\begin{array}{l}10,0 \\
(2,8)\end{array}$ & $\begin{array}{c}1,15 \\
(0,17)\end{array}$ & $\begin{array}{c}0,29 \\
(0,05)\end{array}$ & $\begin{array}{c}0 \\
65 \%\end{array}$ & $\begin{array}{ll}\mathrm{A} & 31 \\
\mathrm{H} & 41 \\
\mathrm{P} & 28\end{array}$ & $\begin{array}{c}1 \\
73 \%\end{array}$ & $\begin{array}{c}1 \\
55 \%\end{array}$ \\
\hline $\mathrm{E}$ & 11 & $\begin{array}{c}2 \\
100 \%\end{array}$ & \begin{tabular}{|lr}
$\mathrm{A}$ & 0 \\
$\mathrm{H}$ & 100 \\
$\mathrm{P}$ & 0
\end{tabular} & $\begin{array}{c}8,8 \\
(1,0)\end{array}$ & $\begin{array}{c}7,5 \\
(1,4)\end{array}$ & $\begin{array}{c}0 \\
100 \%\end{array}$ & $\begin{array}{c}7,5 \\
(1,4)\end{array}$ & $\begin{array}{c}0,98 \\
(0,12)\end{array}$ & $\begin{array}{c}0,31 \\
(0,18)\end{array}$ & $\begin{array}{c}\geq 1 \\
100 \%\end{array}$ & $\begin{array}{ll}\mathrm{A} & 19 \\
\mathrm{H} & 57 \\
\mathrm{P} & 24\end{array}$ & $\begin{array}{c}1 \\
100 \%\end{array}$ & $\begin{array}{c}2 \\
73 \%\end{array}$ \\
\hline $\mathbf{F}$ & 6 & $\begin{array}{c}1 \\
84 \%\end{array}$ & $\begin{array}{lr}\mathrm{A} & 0 \\
\mathrm{H} & 0 \\
\mathrm{P} & 100\end{array}$ & $\begin{array}{c}8,7 \\
(1,5)\end{array}$ & $\begin{array}{c}9,5 \\
(2,1)\end{array}$ & $\begin{array}{c}\geq 1 \\
100 \%\end{array}$ & $\begin{array}{l}10,8 \\
(2,1)\end{array}$ & $\begin{array}{c}1,32 \\
(0,04)\end{array}$ & $\begin{array}{c}0,43 \\
(0,11)\end{array}$ & $\begin{array}{c}0 \text { ou } 1 \\
50 \%\end{array}$ & $\begin{array}{ll}\mathrm{A} & 39 \\
\mathrm{H} & 41 \\
\mathrm{P} & 20\end{array}$ & $\begin{array}{c}2 \\
66 \%\end{array}$ & $\begin{array}{c}1 \text { ou } 2 \\
50 \%\end{array}$ \\
\hline
\end{tabular}

MB1 Age modal en années (fréquence)

SAISMB1 Fréquence (\%) selon les différentes saisons

MBTER Age moyen à la dernière mise bas en années (écart-type)

NMB Nombre moyen de mises bas (écart-type)

PROL Nombre modal de mises bas gémellaires (fréquence)

NNES Nombre moyen d'agneaux mis bas (écart-type)

NNESAN Nombre moyen d'agneaux mis bas par an (écart-type)

NOOAN Nombre moyen de sauts de deux périodes par an (écart-type)

Figure 8. Les différents profils de carrière des brebis, selon les NOOO Nombre modal d'épisodes d'infertilité (fréquence)

DISTRIB Distribution des mises bas selon les saisons $(\%)$ (non compris les premières mises bas)

SAIS Valeur modale de l'indice de saisonnalité et fréquence en $\%$ principaux critères isolés

FERT Valeur modale de l'indice de fertilité et fréquence en $\%$ par l'analvse.

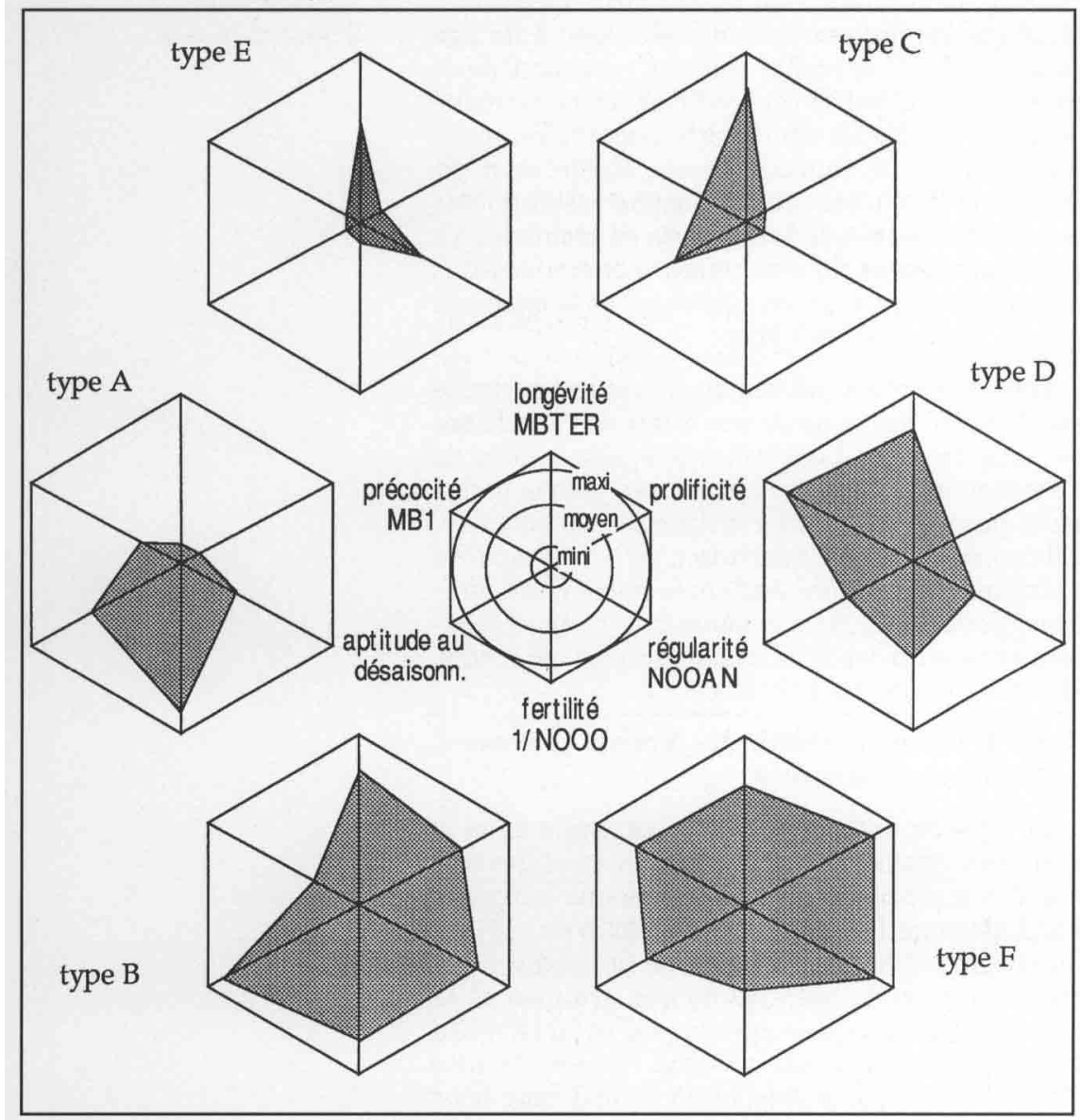

la productivité annuelle. Les brebis de ce type désaisonnent facilement, et régularisent leur rythme de reproduction en sautant fréquemment deux sessions de mise-bas consécutives, comportement particulièrement intéressant chez les bessonnières.

Type D : Des brebis précoces, mais peu prolifiques et moins régulières

Les brebis de ce type sont très comparables à celle du type $\mathrm{F}$ en termes de précocité sexuelle, de longévité et de fertilité. Elles en diffèrent par un niveau de prolificité nettement inférieur, et une capacité plus réduite au désaisonnement (moins de mises-bas d'automne), qui s'accompagne d'une moindre régularité du rythme de reproduction (moins de sauts de deux périodes consécutives). Ceci débouche sur un nombre total d'agneaux nés un peu inférieur, et sur une productivité annuelle plus limitée (1,15 agneau né par an contre 1,32).

Type B : L'archétype de l'adaptation : prolificité, longévité et régularité, pour une productivité record.

Ce type de brebis, qui représente $13 \%$ de l'effectif, occupe le premier rang en termes de longévité, de nombre de mises-bas, de productivité annuelle et de désaisonnement ( $41 \%$ de mises-bas en automne). Il se distingue principalement du type $\mathrm{F}$ par une entrée en reproduction tardive (compensé par une longévité supérieure), une moindre importance des mises-bas d'hiver ( $29 \%$ contre 41 ), une moindre incidence des épisodes d'infertilité, et 
une prolificité un peu inférieure. Il manifeste en revanche une aptitude tout à fait comparable à régulariser son rythme de reproduction en sautant deux périodes de mises-bas consécutives.

Type C : Des brebis tardives et peu prolifiques stabilisant mal leur rythme de reproduction

Les carrières des brebis de ce type (10\% de l'effectif) sont très comparables à celles du type B en ce qui concerne l'âge à la première mise-bas ( 2 ans), la longévité ( 9,3 ans) et la distribution saisonnière des mises-bas (aptitude au désaisonnement). Mais ces brebis moins fertiles stabilisent mal leur rythme de reproduction (très peu de sauts de deux périodes), et font alterner des mises-bas très rapprochées avec des épisodes d'infertilité à répétition. La sanction de cette régulation déficiente est enregistrée au niveau du nombre annuel moyen d'agnelages $(1,13$ contre 1,23), dont la faiblesse est aggravée par l'absence de portées doubles. Au total, le nombre total d'agneaux produits par brebis et surtout la productivité annuelle sont nettement plus faibles que ceux du groupe B.

Type E : Une productivité insuffisante, pour des brebis tardives, peu fécondes et très saisonnées

Ce type de brebis cumule les handicaps : entrée tardive en reproduction, prolificité minimum, faible fertilité, épisodes multiples d'infécondité. Il s'agit manifestement d'animaux mal adaptés au système d'élevage considéré, dont la longévité n'est acquise qu'au prix d'une productivité très faible (moins d'un agneau né par an). Le trait le plus marquant de leur profil de reproduction est probablement la distribution très saisonnée de leurs mises-bas : faible proportion des agnelages d'automne, et concentration extrême sur l'agnelage d'hiver (57\% des mises-bas contre $35 \%$ en moyenne dans les autres types).

\section{3 / Analyse combinée}

La combinaison de l'analyse des performances du troupeau par périodes et de l'analyse des carrières individuelles fournit des outils intéressants pour le diagnostic zootechnique. Nous en présentons quelques exemples ci-dessous.

\section{a / Analyse des rythmes de reproduction}

Le tableau 7, tiré de la matrice (R3), rend compte de la mécanique moyenne des rythmes de reproduction dans le troupeau considéré. Il constitue un modèle par référence auquel il devient possible d'analyser un événement particulier, et de préciser le diagnostic.

Ainsi, le déficit enregistré lors de la mise-bas de l'hiver 1985, consécutive à la lutte d'août 1984 (figure 1) peut-il être essentiellement imputé à la proportion inhabituellement faible des reports de la session de mise-bas du printemps 1984: 13 cas (tableau 4), soit $29 \%$ au lieu de $59 \%$ en moyenne, ceci étant directement consécutif à la proportion exceptionnellement élevée des reports observés de ce même printemps 1984 vers l'automne 1984: 16 cas, soit $36 \%$, contre $7 \%$ en moyenne. Beaucoup de brebis ont donc "redoublé" lors de la lutte de ce printemps 1984, ce qui doit très probablement être mis sur le compte des conditions dans lesquelles s'est déroulée la mise à l'herbe. On peut donc conclure que l'apparente anomalie enregistrée lors de la mise-bas de l'hiver 1985 n'est pas autre chose que la conséquence retardée de ces conditions favorables (ainsi qu'un arrière-effet du profil de fertilité de la campagne 1984-1985, cf. 1.1.).

Autre exemple : le déficit constaté lors de la session de mise-bas d'automne 1983, consécutive à la session de lutte d'avril de la même année (figure 1). Tous les reports depuis les sessions de mise-bas précédentes, de l'hiver 1982 au printemps 1983, sont largement déficitaires (tableau 4). Le diagnostic est donc orienté ou bien sur les conditions d'alimentation durant l'hivernage 19821983, ou plus probablement, compte tenu de l'ampleur du déficit de fertilité, sur le déroulement de la lutte d'avril 1983, comme le suggérait déja l'allure de la courbe de mise-bas correspondante (session d'automne 1983 : figure 2).

\section{b / Interactions entre les comportements individuels des animaux et les variations du milieu d'élevage}

L'étude du déroulement des carrières laisse supposer que, face à la succession des événements qui marquent la vie du troupeau, les animaux qui le composent peuvent avoir des réactions différentes.

Tableau 7. Répartition saisonnière moyenne des mises bas précédant et suivant la mise bas de référence, selon la saison de la mise bas de référence $S$ (pour 100 mises bas en saison de référence). A : Automne - $H$ : Hiver $-P$ : Printemps

\begin{tabular}{|c|c|c|c|c|c|c|c|c|c|c|c|c|}
\hline \multirow{2}{*}{$\begin{array}{c}\text { Mise bas de } \\
\text { référence (i) } \\
\text { Session } \mathrm{S}\end{array}$} & \multicolumn{6}{|c|}{$\begin{array}{l}\text { Mise bas précédente } \\
(\mathrm{i}-1)\end{array}$} & \multicolumn{6}{|c|}{$\begin{array}{l}\text { Mise bas suivante } \\
\qquad(\mathrm{i}+1)\end{array}$} \\
\hline & $S-5$ & $S-4$ & S - 3 & S - 2 & S - 1 & $\begin{array}{c}\text { pas de } \mathrm{MB} \\
\text { antérieure } \\
\grave{a} \mathrm{~S}\end{array}$ & $\mathrm{~S}+1$ & $S+2$ & $\mathrm{~S}+3$ & $\mathrm{~S}+4$ & $S+5$ & $\begin{array}{c}\text { pas de MB } \\
\text { postérieure } \\
\text { à S }\end{array}$ \\
\hline \multirow{4}{*}{$\begin{array}{l}\text { Automne } \\
\text { Hiver } \\
\text { Printemps }\end{array}$} & \multicolumn{2}{|c|}{$\begin{array}{l}\text { épisode } \\
\text { d'infertilité } \\
(000)\end{array}$} & \multirow{2}{*}{\begin{tabular}{|c|}
$\begin{array}{c}\text { saut de } \\
2 \text { sessions } \\
(00)\end{array}$ \\
A 23 \\
\end{tabular}} & \multirow{2}{*}{\begin{tabular}{|c|}
$\begin{array}{c}\text { saut d'une } \\
\text { session } \\
(0)\end{array}$ \\
H 57
\end{tabular}} & \multirow{2}{*}{\begin{tabular}{|c|}
$\begin{array}{c}\text { pas de } \\
\text { saut } \\
\text { (redoublement) }\end{array}$ \\
P 6 \\
\end{tabular}} & \multirow{2}{*}{\begin{tabular}{|c|}
$\begin{array}{c}\text { agnelles } \\
\text { primipares }\end{array}$ \\
9 \\
\end{tabular}} & \multirow{2}{*}{\begin{tabular}{|c|}
$\begin{array}{c}\text { pas de } \\
\text { saut } \\
\text { redodouldementt }\end{array}$ \\
$\mathrm{H} \Sigma$ \\
\end{tabular}} & \multirow{2}{*}{\begin{tabular}{|c|}
$\begin{array}{c}\text { saut dune } \\
\text { session } \\
(0)\end{array}$ \\
P 52 \\
\end{tabular}} & \multirow{2}{*}{\begin{tabular}{|c|}
$\begin{array}{c}\text { saut de } \\
2 \text { sessions } \\
(00)\end{array}$ \\
A 24 \\
\end{tabular}} & \multicolumn{2}{|c|}{$\begin{array}{c}\text { épisode } \\
\text { d'infertilité } \\
(000)\end{array}$} & $\begin{array}{l}\text { mortalité } \\
\text { ou réforme }\end{array}$ \\
\hline & H 3 & P 2 & & & & & & & & H 8 & P 2 & 14 \\
\hline & P 1 & A 7 & H 31 & Р 43 & A $\Sigma$ & 18 & P 0 & A 44 & H 31 & P 7 & A 2 & 17 \\
\hline & A 1 & H 11 & P 14 & A 57 & $\mathrm{H} 0$ & 17 & A 7 & H 59 & P 14 & A 4 & H 1 & 16 \\
\hline
\end{tabular}

L'analyse combinée des carrières individuelles et des performances $d u$ troupeau par période est un outil de diagnostic zootechnique. 
Tableau 8. Comparaison de la réussite de la lutte d'avril 83 pour les différents types de carrières.

\begin{tabular}{|l|c|c|c|}
\hline \multicolumn{1}{|c|}{ Types de carrière } & D et F & A et B & C et E \\
\hline Nombre de brebis réputées fertiles & 16 & 18 & 22 \\
Dont : n'ayant pas agnelé au printemps 83 & 7 & 11 & 18 \\
Nombre de brebis mettant bas à l'automne 83 & 6 & 6 & 3 \\
Nombre d'épisodes d'infertilité incluant la & 0 & 0 & 10 \\
session d'automne 83 & 0 & & \\
\hline
\end{tabular}

La combinaison d'une analyse longitudinale qui permet de caractériser les carrières individuelles des animaux et d'une analyse transversale, qui permet de caractériser les événements constitue une excellente méthode pour étudier ces phénomènes.

Reprenons l'exemple de la session de mise-bas de l'automne 1983, en examinant le comportement des brebis dont l'analyse qui précède a permis de typer la carrière. Le tableau 8 , construit à partir de la matrice (R1), retrace les réactions des brebis qui étaient réputées fertiles lors de la session de lutte correspondante (avril 1983), en fonction de leur type de carrière. Compte tenu de la très faible proportion de brebis qui "redoublent" au printemps (c'est-à-dire qui sont fécondées dès la lutte d'avril après avoir agnelé à la session de mise-bas de printemps immédiatement précédente), nous nous sommes intéressés plus particulièrement aux brebis réputées fertiles lors du début de la lutte d'avril printemps 1983 mais n'ayant pas agnelé juste avant ( $n=36$, seconde ligne du tableau 8 ).

Malgré la faiblesse de l'effectif d'animaux concernés, il est possible de mettre en évidence des différences de comportements selon le type de carrière. Les animaux des types $\mathrm{F}$ et $\mathrm{D}$ apparaissent moins sensibles à la perturbation $(6$ brebis fécondées sur 7) que les brebis des types A et B (6 brebis fécondées sur 11 , les autres "sautant" et se décalant d'une session). Les brebis des types $\mathrm{C}$ et $\mathrm{E}$ manifestent pour leur part des réactions très marquées : très faible fertilité ( 3 mises-bas sur 18 brebis), décalage d'une session ( 5 brebis sur 18 ), et déclenchement de nombreux épisodes d'infertilité (10 brebis). Les différences observées entre types au niveau de la fréquence des mises-bas et au niveau de celle des épisodes d'infertilité sont respectivement significatives aux seuils de 0,05 et de 0,005 .

\section{3 / Discussion}

Compte tenu de l'objectif méthodologique de cet article, de la faiblesse des effectifs étudiés, et de l'incertitude relative à la généralité des phénomènes observés, nous ne nous attacherons pas à la discussion des résultats obtenus, si ce n'est pour souligner la richesse des observations qu'il est possible de mener à partir de données de départ apparemment très frustes.

En ce qui concerne la méthode elle-même, diverses améliorations sont envisageables. Il apparaît par exemple souhaitable de construire des indices de fertilité et de désaisonnement plus pertinents que ceux que nous avons utilisés. Par la suite, on peut penser pour cette méthode à divers types d'applications. Une meilleure compréhension du déroulement des carrières femelles dans des systèmes d'élevage variés déboucherait à peu de frais sur des propositions opératoires en termes de conduite du troupeau et peut-être de choix du matériel génétique. Ainsi, il apparaît que la longévité ne peut à elle seule suffire comme indicateur d'adaptation des animaux à un système d'élevage donné : elle doit être associée à des indicateurs de productivité. La classification des carrières issue de l'AFC, même si elle n'a qu'un pouvoir explicatif limité (32\% de la variance totale expliquée par les trois premiers axes et $63 \%$ des individus classés), apporte à cet égard des éléments d'interprétation originaux.

L'analyse présentée ci-dessus a par exemple permis de mieux comprendre la manière particulière dont s'élabore la production d'agneaux dans le système d'élevage étudié, et de préciser deux points :

- le rôle déterminant, pour la régularité de la production du troupeau (facteur essentiel pour la maîtrise technique de la gestion de l'exploitation), de la réussite de la lutte de printemps, principale source de la variabilité constatée au niveau des rythmes de reproduction. Ceci n'apparaissait pas évident dans un système de production si peu intensifié

- l'interprétation zootechnique des sauts de deux sessions consécutives de reproduction (00), qui apparaissent nettement, dans ce contexte, comme un mécanisme de régulation positivement lié à la productivité. La conjonction a priori paradoxale de l'aptitude à réaliser de tels sauts et de l'aptitude au désaisonnement semble constituer l'une des clés de l'adaptation des brebis au système étudié, dont la logique de fonctionnement est loin de répondre à l'objectif productiviste classique des "trois mises-bas en deux ans". L'éleveur cherche plutôt, à travers la multiplication des sessions de lutte, à régulariser la production du troupeau et à minimiser les risques liés à chaque session, en offrant aux animaux de multiples possibilités de "rattrapage" et en permettant l'expression d'aptitudes individuelles variées.

Cependant, une valorisation complète de ces analyses nécessiterait le recueil d'informations complémentaires permettant d'affiner le diagnostic relatif à la nature des perturbations subies par 
le troupeau. Les enquêtes rétrospectives s'avérant peu fiables, dès lors que l'on souhaite remonter plus de quelques années en arrière, l'idéal serait que les éleveurs enregistrent au fur et à mesure quelques commentaires relatifs à l'alimentation du troupeau et aux conditions dans lesquelles se déroulent les sessions de lutte et de mise-bas. La réalisation d'un inventaire régulier du troupeau représente à notre avis une contrainte nettement plus lourde pour les éleveurs. Ces inventaires sont cependant mis en œuvre dans tous les programmes de contrôle des performances (les dénombrements réalisés annuellement par les éleveurs qui bénéficient des primes à la brebis ne sont pratiquement pas utilisables à des fins d'analyse zootechniques). Dans les exploitations concernées, le suivi des pratiques des éleveurs en matière de conduite des troupeaux (allotement, alimentation, conduite au pâturage, reproduction, conduite sanitaire, politique de réforme, etc) permettrait de préciser les relations existant entre ces pratiques et les performances des animaux, et fournir les clés d'interprétation pour les enregistrements beaucoup plus légers qui sont réalisés dans la majorité des élevages. Bien des élevages ovins resteront en effet sans doute encore longtemps en dehors des réseaux de fermes de référence, et il importe, dans une perspective d'appui technique aux exploitants, de valoriser au mieux l'information qui y est disponible, et qui est principalement contenue dans les carnets d'agnelage.

La principale limite à laquelle se heurte ce type de travail est inhérente au grand recul dans le temps qu'il suppose. Le nombre d'années de recul nécessaire (A) pour obtenir un échantillon non biaisé de $\mathrm{N}$ carrières complètes peut être estimé comme suit, dans l'hypothèse ou l'effectif des agnelles entrant en reproduction chaque année (n) est à peu près constant :

$$
\mathrm{A}=\operatorname{Max}(\mathrm{VPROD})+\mathrm{N} / \mathrm{n}+\mathrm{r}-1
$$

avec $\mathbf{r}=$ durée de la période de recul nécessaire pour pouvoir décider qu'une brebis est sortie du troupeau (dans l'exemple étudié, $r=2$ années et $\mathrm{A}=13$ années : un recul supplémentaire de 3 ans aurait permis d'obtenir au moins 200 carrières complètes).

Bien entendu, la réalisation d'une telle analyse est strictement conditionnée par la disponibilité des anciens carnets d'agnelage, que les éleveurs ne conservent pas toujours sur des durées aussi longues. Une information sur ce point serait nécessaire, si l'on entendait vulgariser ce type de valorisation de l'information contenue dans ces carnets. Dans cette perspective, nous envisageons la construction d'un logiciel permettant d'automatiser les principaux calculs.

Toutefois, il ne semble pas exclu de pouvoir travailler dans l'avenir sur des durées beaucoup plus courtes, mais les méthodes nécessaires devront au préalable être testées et validées à partir d'études portant sur des échantillons non biaisés de carrières complètes.

\section{Conclusion}

Il ne paraît pas illusoire, au terme de cette étude, d'envisager d'utiliser beaucoup plus intensivement qu'aujourd'hui l'information contenue dans les carnets d'agnelage, information dont le recueil par l'éleveur n'est pas très contraignant, et se justifie par ailleurs sur le plan pratique. La constitution d'une base de données reprenant rétrospectivement cette information permet de caractériser finement l'évolution des performances de reproduction du troupeau considéré au cours du temps, et de la mettre en relation, à des fins de diagnostic, avec les évènements divers qui ont marqué la vie du troupeau. Elle permet également d'informer le dossier jusqu'ici trop peu étudié des carrières animales. De telles bases de données pourraient se prêter à diverses simulations, comme le test de l'intérêt d'une politique de réforme des brebis infertiles ou de méthodes améliorant la maitrise de la fertilité à la lutte de printemps.

Ce type d'analyse met en évidence la complémentarité des études menées à l'échelle du troupeau et à l'échelle des individus, et souligne l'intérêt d'adopter des pas de temps longs pour analyser le fonctionnement des systèmes d'élevage.

\section{Remerciements}

Nous remercions Ph. Vissac, en compagnie de qui nous avons initié ce travail, qui s'est prolongé bien au-delà de son stage. Tous nos remerciements également à Monsieur B. Gassend, qui a bien voulu accueillir cette recherche et mettre à notre disposition les enregistrements qu'il a réalisés avec tant de constance et de soin dans son élevage, ainsi qu'aux lecteurs qui nous ont aidé à améliorer ce texte. 


\section{Références bibliographiques}

BAKER R.L., STEINE T.A., VABENE A.W., BEKKEN A., GJEDREM T., 1978. Effect of mating ewe lambs on lifetime productive performance. Acta Agriculturae Scandinavica, vol. 28 (2) : 203-217.

COULON J.B., LANDAIS E., GAREL J.P., 1990. Pathologie et productivité de la vache laitière : interrelations à l'échelle de la lactation. Ann. Rech.Vet. 20(4) : 431-442.

DALTON D.C., BIGHAM M.L., SUMMER R.M.W., 1976. A comparison between the Romney and Merino x Romney on hill country. New Zealand journal of Experimental Agriculture, vol $4: 27-33$.

ESCOUFIER Y. et al 1987. LEAS, Logiciel d'Enseignement et d'Analyse Scientifique. Montpellier, INRA, Laboratoire de Biomètrie, $316 p$

FLAMANT J.C., BIBE B., GIBON A., VU-TIEN-KHANG $\mathrm{J}, 1979$. Approche pour l'amélioration génétique des races locales : notion de rusticité. Paris, INRA-ITOVIC, 5è journées de la recherche ovine et caprine, pp. 427-447.

GUNN R.G., 1977. The effects of two nutritional environments from 6 weeks prepartum to 12 months of age on lifetime performance and reproduction potential of Scottish blackface ewes in two adult environments. Animal Production, 1977, 25(2) : 155-164.
HARESIGN W., 1983. Underfeeding and reproduction : physiological mecanims. In : Reproduction des ruminants en zone tropicale. Pointe-à-Pître (Guadeloupe), 8-10 juin 1983. Versailles, INRA Publications, coll. Les colloques de l'INRA, n 20 (1984) : 339-365.

RICORDEAU G., FLAMANT J.C., 1969. Croisement entre les races Préalpes du Sud et Frisonne. IIReproduction, viabilité, croissance, conformation. Ann. Zoot., 18 (2) : 131-149.

VALLERAND F., 1979. Réflexions sur l'utilisation des races locales en élevage africain. Toulouse, Thèse Inst. Polytech./INRA , 242 p. + annexes.

VALLERAND F., SANTUCCI P.M., 1987. Conduite des animaux et équilibration des systèmes fourragers très saisonnés. In : Pâturage et alimentation des ruminants en zone tropicale humide. Pointe-à-Pître (Guadeloupe), 2 6 juin 1987. Paris, INRA Publications, 1989, pp.259-296.

VALLERAND F. 1988. La rusticité, niveau et méthodes d'approche en milieu réel. In : Hubert B., Girault N. éd. : De la touffe d'herbe au paysage, Actes du séminaire de Viens (France), 13-14 janvier 1983. Versailles, INRA$\mathrm{SAD}: 85-101$

VISSAC P., 1988. Approche dynamique des performances de reproduction de troupeaux ovins; liaisons avec les pratiques d'élevage. Mémoire de fin d'études, Dijon, ENSAA, 47 p. + annexes.

\section{Summary}

Better exploitation of lambing data to evaluate reproductive performance on a meat production sheep farm.

Using, an example, a study conducted on a meat production sheep farm in southern France, the authors propose a method for analyzing, retrospectively, breeding performance on the basis of information recorded in lambing registers, a type of record widely used by French sheep farmers.

Three complementary investigation approaches may be developed by organizing this information in different ways :

- an analysis of seasonal and between-year variation of breeding performance at flock level ;

- an analysis of within-flock variability of individual performance (precocity, aptitude for out-of- season breeding, regularity, prolificacy, longevity) resulting in an overall characterization of ewe lifetime performance ;

- a combined analysis associating the above approaches : the dynamics of production cycles can be calculated from this. These are the result of interactions between variations in husbandry and production the conditions and individual sheep behaviour.

Developing this type of investigation and applying it to a diversity of sheep production systems could produce operational results for flock management and possibly also for selecting appropriate genetic material.

LASSEUR J., LANDAIS E., 1992. Mieux valoriser l'information contenue dans les carnets d'agnelage pour évaluer des performances et des carrières de reproduction en élevage ovin-viande. INRA Prod. Anim., 5 (1) 43- 58. 